\title{
Evaluation of DNA damage in white blood cells of healthy human volunteers using the alkaline comet assay and the chromosome aberration test
}

\author{
Nevenka Kopjar», Davor Želježić and Verica Garaj-Vrhovac \\ Institute for Medical Research and Occupational Health, Mutagenesis Unit Zagreb, Croatia; \\ 凶e-mail:nkopjar@imi.hr
}

Received: 16 January, 2006; revised: 16 Febraury, 2006; accepted: 23 February, 2006

avaiable on-line: 03 April, 2006

\begin{abstract}
The present study was undertaken to contribute to the characterization of the degree of variability in baseline damage in white blood cells from control population, and to investigate how this variability is associated with external and internal factors. Altogether $\mathbf{1 7 0}$ healthy volunteers, randomly selected from the general population of the Republic of Croatia, participated in the study. Two sensitive tests: the alkaline comet assay and the chromosome aberration test were applied to study the background levels of DNA damage in their white blood cells. The results point to inter-individual differences, indicating different genome sensitivity. As revealed by both assays, the background levels of DNA damage were mostly influenced by smoking habit as well as medical exposure (especially to diagnostic X-rays). Sex and age of subjects did not significantly influence the values of DNA damage recorded in the white blood cells. Although higher levels of DNA damage were recorded in blood samples collected during winter and autumn, they were mostly influenced by medicinal exposure and smoking habit. Statistical evaluation of the data confirmed that a positive correlation exists between DNA migration and the number of long-tailed nuclei found with the comet assay and the total number of chromosome aberrations. The data obtained can serve as control values in forthcoming biomonitoring studies.
\end{abstract}

Keywords: white blood cells, peripheral blood, DNA damage, alkaline comet assay, lymphocytes, chromosome aberration test

The actual values in genotoxicological monitoring of a human population can partly be influenced by endogenous factors including those of biological origins such as gender, age and white blood cell count. A variety of external factors such as chemicals, physical agents, viruses, life-style (smoking and drinking habits, nutrition), residential and/or working areas and seasonal changes can also influence the levels of genetic damage in white blood cells of individuals (Major et al., 1998).

Knowledge of the normal levels of genetic abnormalities in the general population is essential before information on the levels of similar genetic abnormalities in subjects exposed to potentially genotoxic agents in the work environment can be properly interpreted (Anderson et al., 1988).
Human biomonitoring can be performed using different genetic markers. Chromosomal aberrations (CA) in peripheral blood lymphocytes (PBL) as well as other cytogenetic biomarkers such as sister chromatid exchange (SCE) and micronuclei (MN), have been used for a relatively long time in surveillance of work environments with low-dose exposures to mutagens or carcinogens. Peripheral blood lymphocytes, because of their easy availability, have been traditionally used to monitor the effects of exposure to known or suspected mutagens. This methodology is very well established, and criteria to be used in such studies are well documented (Natarajan et al., 1996; Albertini et al., 2000). It has often been postulated that cytogenetic tests may also serve as biomarkers of an early mutagen effect indicating increased cancer risk (Hagmar et al., 1998). 
During the last decade, the single cell gel electrophoresis (SCGE) or comet assay was introduced as a rapid, sensitive and inexpensive technique for qualitative and quantitative assessment of DNA damage in single cells (Rojas et al., 1999; Møller et al., 2000; Singh, 2000; Tice, 2000; Collins, 2004). While biomonitoring studies employing cytogenetic techniques are limited to circulating lymphocytes and involve proliferating cell populations, the comet assay can be applied to proliferating and non-proliferating cells (Kassie et al., 2000). This method, within a short time, has found wide usage in epidemiological and biomonitoring studies in humans, to determine DNA damage, as a result of endogenous factors and lifestyle (Betti et al., 1994; Hellman et al., 1997; Frenzilli et al., 1997; Bajpayee et al., 2002; Morillas et al., 2002; Møller et al., 2002; Speit et al., 2003; Mastaloudis et al., 2004; Hininger et al., 2004; Hoffman \& Speit, 2005) as well as due to occupational exposure (Awara et al., 1998; Şardaş et al., 1998; Wojewódzka et al., 1998; Andreoli et al., 1999; Palus et al., 1999; Pitarque et al., 1999; Somorovská et al., 1999; Zhu et al., 1999, 2001; Moretti et al., 2000; Kopjar \& Garaj-Vrhovac, 2001; Maluf et al., 2001; Želježić \& Garaj-Vrhovac, 2001; GarajVrhovac \& Kopjar, 2003) as well as environmental exposures (Šrám et al., 1998; Hellman et al., 1999; Valverde et al., 1999).

The accuracy of any risk assessment, especially in the case of low doses, depends upon both the resolution of the methods being used, and the baseline data obtained in well-selected controls. The use of several, independent end-points improves the resolution of an investigation and provides a more accurate risk assessment (Major et al., 1998).

Here we report data obtained by a two-biomarker approach on peripheral blood samples collected from healthy subjects randomly selected from the general population of the Republic of Croatia. The present study was undertaken to contribute to the characterisation of the degree of variability in baseline damage in white blood cells, to investigate how this variability is associated with external and internal factors, and to help to establish background data which may be of use as control values in future biomonitoring studies.

\section{MATERIALS AND METHODS}

Population characteristics. The population under study consisted of 170 blood donors (76 female and 94 male; average age $35 \pm 10$, age range: 20 to 64 years). They were of mixed social class and occupation (students, teachers, housewives, administrative employees, blue collar workers and unemployed). Most of them donated their blood samples during pre-employment medical check-ups, while others were freely engaged in the study.

The investigation was performed in accordance with high standards of ethics. Informed consent was obtained from all participating subjects prior to the start of the study. Blood donors were also informed about the aim and the experimental details of the study. All of them were healthy at the moment of blood sampling and interviews. Each participant completed a standardised questionnaire, designed to obtain relevant details of current health status, health history and lifestyle. Data on exposure to possible confounding factors: smoking habits, alcohol consumption, medicinal usage, contraception, severe infections or viral diseases during the last six months, recent vaccinations, presence of known inherited genetic disorders and chronic diseases, family history of cancer, exposure to indoor/outdoor pollutants, sunlight exposure, exposure to diagnostic X-rays, plus previous radio- or chemotherapy were collected. Donors who reported alcohol consumption, history of cancer and any cancer therapy, known inherited genetic disorders, treated acute infections, and/or chronic non-infectious diseases, intake of vitamins or intensive sportive activities during the last week were excluded from this study.

Seventy-six subjects were regular smokers (29 female and 47 male subjects), and 94 were nonsmokers (47 female and 47 male). One month prior to the blood sampling, 52 subjects were subjected to diagnostic chest $\mathrm{X}$-rays or dental X-rays, usualy during pre-employment medical checkups; two subjects reported medical exposure to magnetic resonance imaging (MRI) a few months ago, while two reported occasional use of antirheumatic drugs through one year prior to the study. Other subjects reported no medicinal treatments. Their dietary habits were not appreciably different. None of them had history of occupational exposure to known genotoxic chemicals.

Blood sampling. Peripheral blood samples (V $=5 \mathrm{ml}$ ) were collected under sterile conditions by venipuncture into heparinised tubes (BD vacutainer, Becton Dickinson, NJ, USA) in the morning hours, between 9 and 10 a.m. After collection, all blood samples were randomly coded, cooled at $4^{\circ} \mathrm{C}$, transported to the laboratory and processed as quickly as possible (usually within $2 \mathrm{~h}$ following the blood sampling).

The alkaline comet assay. The comet assay was carried out under alkaline conditions, basically as described by Singh et al. (1988). Two fully-frosted microscopic slides per subject were prepared. Each slide was covered with $1 \%$ normal melting point (NMP) agarose (Sigma). After solidification, the gel was scraped off from the slide. The slides were then coated with $0.6 \%$ NMP agarose. When this layer 
had solidified a second layer containing a whole blood sample $(4 \mu \mathrm{l})$ mixed with $0.5 \%$ low melting point (LMP) agarose (Sigma) was placed on the slides. After $10 \mathrm{~min}$ of solidification on ice, slides were covered with $0.5 \%$ LMP agarose. Afterwards the slides were immersed for at least $1 \mathrm{~h}$ in ice-cold freshly prepared lysing solution $(2.5 \mathrm{M} \mathrm{NaCl}, 100$ $\mathrm{mM} \mathrm{Na}{ }_{2}$ EDTA, $10 \mathrm{mM}$ Tris/HCl, $1 \%$ Na-sarcosinate (Sigma), pH 10, with 1\% Triton X-100 (Sigma) and $10 \%$ dimethyl sulfoxide (Kemika) added fresh to lyse cells and allow DNA unfolding. The slides were then randomly placed side by side in a horizontal gel-electrophoresis tank, facing the anode. The unit was filled with freshly prepared electrophoretic buffer (300 mM NaOH, $1 \mathrm{mM} \mathrm{Na}{ }_{2}$ EDTA, $\left.\mathrm{pH} 13.0\right)$ and the slides were set in this alkaline buffer for $20 \mathrm{~min}$ to allow DNA unwinding and expression of alkalilabile sites. Denaturation and electrophoresis were performed at $4^{\circ} \mathrm{C}$ under dim light. Electrophoresis was carried out for the next $20 \mathrm{~min}$ at $25 \mathrm{~V}$ (300 $\mathrm{mA}$ ). After electrophoresis the slides were washed gently three times at 5-min intervals with a neutralisation buffer $(0.4 \mathrm{M}$ Tris/ $\mathrm{HCl}, \mathrm{pH} 7.5)$ to remove excess alkali and detergents. Each slide was stained with ethidium bromide $(20 \mu \mathrm{g} / \mathrm{ml})$ and covered with a coverslip. Slides were stored at $4^{\circ} \mathrm{C}$ in humidified sealed containers until analysis. To prevent additional DNA damage, handling of blood samples and all steps included in the preparation of slides for the comet analysis were conducted under yellow light or in the dark. Furthermore, to avoid possible position effects during electrophoresis, each replicate was processed in a different electrophoretic run.

Slides were examined at 250× magnification with a fluorescence microscope (Zeiss, Germany), equipped with an excitation filter of $515-560 \mathrm{~nm}$ and a barrier filter of $590 \mathrm{~nm}$. A total of 100 comets per subject were scored (50 from each of two replicate slides). Comets were randomly captured at a constant depth of the gel, avoiding the edges of the gel, occasional dead cells and superimposed comets. The microscope was connected to a black and white camera with a computer-based image analysis system (Comet Assay II, Perceptive Instruments Ltd., UK). This image analysis system acquires images, computes the integrated intensity profiles for each cell, estimates the comet cell components, and then evaluates the range of derived parameters. As a measure of DNA damage in this study tail length was used. It was calculated from the centre of the head and presented in micrometers.

Chromosome aberration (CA) test. In order to rigorously control technical conditions, all materials were purchased as single batches prior to the start of the study. The chromosome aberration test was performed in agreement with current IPCH and IAEA guidelines (Albertini et al., 2000; IAEA,
2001). Blood samples were cultivated in Ham's F-10 medium (Sigma) supplemented with $20 \%$ fetal calf serum, phytohaemagglutinin, and antibiotics penicillin and streptomycin. Duplicate cultures per subject were set up and incubated at $37 \pm 1^{\circ} \mathrm{C}$ for $48 \mathrm{~h}$. To arrest dividing lymphocytes in metaphase, colchicine $(0.004 \%)$ was added $3 \mathrm{~h}$ prior to the harvest. Cultures were centrifuged at 1000 r.p.m. for $10 \mathrm{~min}$, the supernatant was carefully removed, and the cells were resuspended in a hypotonic solution $(0.075 \mathrm{M}$ $\mathrm{KCl}$ ) at $37^{\circ} \mathrm{C}$. After centrifugation for $10 \mathrm{~min}$ at 1000 r.p.m., the cells were fixed with a freshly prepared fixative of ice cold methanol/glacial acetic acid (3:1, $\mathrm{v} / \mathrm{v})$. Fixation and centrifugation were repeated several times until the supernatants were clear. Cells were pelleted and resuspended in a minimal amount of fresh fixative to obtain a homogeneous suspension. The cell suspension was dropped onto microscope slides and left to air-dry. Slides were stained with 5\% Giemsa solution (Sigma). All slides were coded and scored blindly. Two hundred metaphases per subject (100 metaphases from each parallel culture) were analysed for chromosomal aberrations. Structural chromosome aberrations were classified based on the number of sister chromatids and breakage events involved. Only metaphases containing 45-47 centromeres were analysed. Total numbers and types of aberrations, as well as the percentage of aberrant cells per each subject were evaluated.

Statistical analyses. Statistical analyses were carried out using Statistica 5.0 package (StatSoft, Tulsa, USA). Each subject was characterized for the extent of DNA damage by considering the mean ( \pm standard deviation), median, range and dispersion coefficient $(\mathrm{H})$ for the comet tail lengths measured. The dispersion coefficient was calculated as the ratio of the sample variance to the sample mean (Albertini et al., 2000). Moreover, cells were classified as either "undamaged" or "damaged" by considering threshold levels indicating the comets with a longtailed nucleus (LTN), i.e. the length over the 95th percentile of the distribution of the tail lengths (Betti et al., 1994; Moretti et al., 2000). Multiple comparisons between groups were done by means of multifactor ANOVA with post-hoc Scheffé test as well as using the canonical analysis on the logarithmic transformed data. The level of statistical significance was set at $P<0.05$.

\section{RESULTS}

\section{The alkaline comet assay}

Characteristics of the subjects: age, sex, smoking habits, medical exposure, time of the blood sampling, and individual DNA damage data are report- 
Table 1. Demographic data of healthy human volunteers and results of alkaline comet assay and chromosome aberration (CA) analysis in their white blood cells

\begin{tabular}{|c|c|c|c|c|c|c|c|c|c|c|c|c|c|c|}
\hline \multirow{2}{*}{ 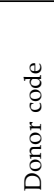 } & \multirow{2}{*}{$\begin{array}{l}\text { Sex } \\
\text { age }\end{array}$} & \multirow{2}{*}{$\begin{array}{l}\text { Smoking } \\
\text { habit } \\
\text { Medical } \\
\text { exposure }\end{array}$} & \multirow{2}{*}{$\begin{array}{l}\text { Sample } \\
\text { Month }\end{array}$} & \multicolumn{5}{|c|}{ DNA migration - comet tail length $(\mu \mathrm{m})$} & \multicolumn{5}{|c|}{$\begin{array}{l}\text { Total number and dis- } \\
\text { tribution of CA }\end{array}$} & \multirow{2}{*}{$\begin{array}{l}\text { Cells } \\
\text { with } \\
\text { CA } \\
(\%)\end{array}$} \\
\hline & & & & $\begin{array}{l}\text { Mean } \\
\pm \text { S.D. }\end{array}$ & Med. & $\mathrm{H}$ & Min. & Max. & $\begin{array}{l}\text { of } \\
\text { LTN }\end{array}$ & $\mathrm{B}_{1}$ & $\mathrm{~B}_{2}$ & Ac & $\Sigma$ & \\
\hline 1 & $\mathrm{M} / 20$ & $S / X$ & 10 & $14.17 \pm 1.03$ & 14.26 & 0.10 & 11.67 & 16.86 & 2 & 0 & 2 & 0 & 2 & 1.0 \\
\hline 2 & $\mathrm{M} / 20$ & $S$ & 2 & $14.38 \pm 1.49$ & 14.42 & 0.15 & 11.54 & 17.95 & 5 & 0 & 0 & 0 & 0 & 0 \\
\hline 3 & $\mathrm{M} / 21$ & NS & 1 & $12.65 \pm 0.92$ & 12.97 & 0.07 & 11.02 & 15.56 & 0 & 0 & 0 & 0 & 0 & 0 \\
\hline 4 & F / 21 & NS / X & 1 & $13.70 \pm 0.94$ & 13.61 & 0.07 & 11.67 & 16.86 & 2 & 0 & 0 & 0 & 0 & 0 \\
\hline 5 & $\mathrm{~F} / 21$ & $S$ & 12 & $13.56 \pm 1.10$ & 13.61 & 0.09 & 11.67 & 16.21 & 0 & 0 & 0 & 1 & 1 & 0.5 \\
\hline 6 & $\mathrm{M} / 21$ & S & 10 & $14.53 \pm 0.75$ & 14.26 & 0.04 & 12.97 & 16.21 & 0 & 0 & 0 & 0 & 0 & 0 \\
\hline 7 & M / 21 & $S / X$ & 10 & $14.05 \pm 1.15$ & 14.26 & 0.09 & 11.02 & 15.66 & 0 & 1 & 0 & 0 & 1 & 0.5 \\
\hline 8 & M / 21 & NS / $\mathrm{X}$ & 2 & $14.90 \pm 1.28$ & 14.74 & 0.11 & 12.18 & 17.31 & 4 & 1 & 0 & 0 & 1 & 0.5 \\
\hline 9 & M / 22 & NS & 1 & $13.89 \pm 1.34$ & 14.26 & 0.13 & 9.08 & 16.21 & 0 & 0 & 0 & 0 & 0 & 0 \\
\hline 10 & M / 22 & NS / X & 2 & $14.23 \pm 1.34$ & 14.42 & 0.13 & 11.54 & 17.31 & 1 & 1 & 0 & 0 & 1 & 0.5 \\
\hline 11 & M / 22 & NS & 12 & $14.03 \pm 1.65$ & 13.46 & 0.19 & 11.54 & 19.87 & 9 & 0 & 0 & 0 & 0 & 0 \\
\hline 12 & M / 22 & NS / X & 2 & $14.62 \pm 1.34$ & 14.74 & 0.12 & 11.54 & 17.31 & 3 & 0 & 0 & 0 & 0 & 0 \\
\hline 13 & M / 23 & $S$ & 2 & $15.55 \pm 1.34$ & 15.38 & 0.12 & 12.18 & 20.51 & 11 & 1 & 0 & 0 & 1 & 0.5 \\
\hline 14 & $\mathrm{~F} / 23$ & NS & 3 & $14.72 \pm 1.24$ & 14.91 & 0.11 & 11.02 & 16.86 & 2 & 2 & 0 & 0 & 2 & 1.0 \\
\hline 15 & M / 23 & NS & 5 & $13.02 \pm 0.94$ & 12.97 & 0.07 & 11.02 & 14.91 & 0 & 0 & 0 & 0 & 0 & 0 \\
\hline 16 & M / 23 & NS / X & 10 & $13.78 \pm 0.79$ & 13.61 & 0.05 & 12.32 & 14.91 & 0 & 0 & 1 & 0 & 1 & 0.5 \\
\hline 17 & M / 23 & $S / X$ & 2 & $15.29 \pm 1.75$ & 15.38 & 0.20 & 10.90 & 19.23 & 19 & 0 & 0 & 0 & 0 & 0 \\
\hline 18 & M / 23 & NS / X & 2 & $13.51 \pm 1.49$ & 13.46 & 0.16 & 10.26 & 17.95 & 1 & 0 & 1 & 0 & 1 & 0.5 \\
\hline 19 & $\mathrm{M} / 24$ & $\mathrm{NS} / \mathrm{X}$ & 3 & $15.28 \pm 1.64$ & 15.38 & 0.18 & 10.90 & 19.23 & 14 & 1 & 0 & 0 & 1 & 0.5 \\
\hline 20 & M / 24 & NS / X & 3 & $15.38 \pm 1.85$ & 15.38 & 0.22 & 10.26 & 20.51 & 21 & 2 & 0 & 0 & 2 & 1.0 \\
\hline 21 & M / 24 & NS & 1 & $14.15 \pm 0.92$ & 14.26 & 0.06 & 12.32 & 17.50 & 2 & 0 & 0 & 0 & 0 & 0 \\
\hline 22 & M / 24 & $S / X$ & 10 & $15.04 \pm 0.94$ & 15.56 & 0.06 & 12.32 & 16.21 & 0 & 0 & 0 & 1 & 1 & 0.5 \\
\hline 23 & F / 24 & NS & 7 & $13.03 \pm 1.25$ & 12.82 & 0.12 & 10.90 & 16.67 & 0 & 1 & 0 & 0 & 1 & 0.5 \\
\hline 24 & $\mathrm{M} / 25$ & $S / X$ & 3 & $15.38 \pm 1.92$ & 15.38 & 0.24 & 10.90 & 19.87 & 18 & 1 & 0 & 0 & 1 & 0.5 \\
\hline 25 & F / 25 & $S / X$ & 3 & $15.35 \pm 1.54$ & 15.38 & 0.15 & 11.54 & 19.87 & 12 & 0 & 0 & 0 & 0 & 0 \\
\hline 26 & M / 25 & NS & 9 & $12.54 \pm 1.13$ & 12.32 & 0.10 & 10.37 & 14.26 & 0 & 0 & 0 & 1 & 1 & 0.5 \\
\hline 27 & M / 25 & NS / X & 10 & $14.48 \pm 1.21$ & 14.26 & 0.10 & 11.02 & 16.86 & 4 & 1 & 0 & 1 & 2 & 1.0 \\
\hline 28 & F / 25 & NS & 5 & $12.52 \pm 0.84$ & 12.64 & 0.06 & 9.72 & 13.61 & 0 & 1 & 0 & 0 & 1 & 0.5 \\
\hline 29 & F / 25 & NS & 5 & $12.75 \pm 0.84$ & 12.97 & 0.06 & 11.02 & 13.61 & 0 & 0 & 0 & 0 & 0 & 0 \\
\hline 30 & $\mathrm{M} / 25$ & $S$ & 10 & $13.98 \pm 1.04$ & 13.94 & 0.08 & 12.32 & 16.21 & 0 & 2 & 0 & 0 & 2 & 1.0 \\
\hline 31 & F / 25 & NS & 6 & $13.70 \pm 1.43$ & 13.46 & 0.15 & 10.26 & 17.31 & 1 & 0 & 0 & 0 & 0 & 0 \\
\hline 32 & F / 25 & NS & 7 & $13.98 \pm 1.55$ & 14.10 & 0.17 & 10.26 & 17.31 & 2 & 0 & 0 & 0 & 0 & 0 \\
\hline 33 & F / 26 & $S / X$ & 3 & $15.91 \pm 2.24$ & 16.03 & 0.31 & 10.26 & 19.87 & 35 & 0 & 0 & 1 & 1 & 0.5 \\
\hline 34 & M / 26 & $S / X$ & 3 & $15.21 \pm 1.73$ & 15.38 & 0.20 & 11.54 & 19.23 & 15 & 2 & 0 & 0 & 2 & 1.0 \\
\hline 35 & M / 26 & $S$ & 10 & $13.64 \pm 0.97$ & 13.61 & 0.07 & 12.32 & 15.56 & 0 & 2 & 0 & 0 & 2 & \\
\hline 36 & $\mathrm{M} / 26$ & $S / X$ & 10 & $13.82 \pm 1.47$ & 13.61 & 0.16 & 11.02 & 16.86 & 2 & 1 & 0 & 0 & 1 & 0.5 \\
\hline 37 & M / 26 & NS & 3 & $14.60 \pm 0.76$ & 14.91 & 0.04 & 12.32 & 15.56 & 0 & 0 & 2 & 0 & 2 & 1.0 \\
\hline 38 & M / 26 & NS & 9 & $12.63 \pm 1.34$ & 12.64 & 0.14 & 10.37 & 14.91 & 0 & 0 & 0 & 0 & 0 & 0 \\
\hline 39 & F / 26 & $S$ & 11 & $15.04 \pm 0.98$ & 14.91 & 0.06 & 12.97 & 17.50 & 4 & 0 & 0 & 0 & 0 & 0 \\
\hline 40 & M / 26 & NS & 5 & $12.45 \pm 0.82$ & 12.32 & 0.05 & 10.37 & 14.26 & 0 & 0 & 0 & 0 & 0 & 0 \\
\hline 41 & M / 26 & S & 10 & $15.00 \pm 1.17$ & 14.91 & 0.09 & 12.32 & 16.86 & 6 & 2 & 0 & 0 & 2 & 1.0 \\
\hline 42 & M / 26 & NS / X & 4 & $14.96 \pm 1.49$ & 14.74 & 0.15 & 10.90 & 18.59 & 10 & 1 & 0 & 0 & 1 & 0.5 \\
\hline
\end{tabular}




\begin{tabular}{|c|c|c|c|c|c|c|c|c|c|c|c|c|c|c|}
\hline 43 & M / 26 & $S / X$ & 4 & $14.44 \pm 1.82$ & 14.74 & 0.23 & 10.90 & 18.59 & 7 & 1 & 0 & 0 & 1 & 0.5 \\
\hline 44 & M / 27 & NS / X & 3 & $14.98 \pm 2.02$ & 15.06 & 0.31 & 9.62 & 18.59 & 16 & 0 & 0 & 0 & 0 & 0 \\
\hline 45 & M / 27 & NS & 5 & $12.84 \pm 0.75$ & 12.97 & 0.04 & 11.02 & 13.61 & 0 & 1 & 0 & 0 & 1 & 0.5 \\
\hline 46 & M / 27 & NS / X & 3 & $15.07 \pm 1.18$ & 14.91 & 0.09 & 12.32 & 17.50 & 14 & 1 & 0 & 1 & 2 & 1.0 \\
\hline 47 & M / 27 & NS & 5 & $12.43 \pm 0.76$ & 12.32 & 0.05 & 11.02 & 13.61 & 0 & 0 & 0 & 0 & 0 & 0 \\
\hline 48 & M / 27 & NS / X & 2 & $14.62 \pm 1.60$ & 14.74 & 0.17 & 10.26 & 17.95 & 6 & 1 & 0 & 0 & 1 & 0.5 \\
\hline 49 & F / 27 & S & 7 & $13.31 \pm 1.43$ & 13.46 & 0.16 & 9.62 & 16.67 & 0 & 0 & 0 & 0 & 0 & 0 \\
\hline 50 & M / 28 & $S / X$ & 2 & $15.33 \pm 1.43$ & 15.38 & 0.13 & 11.54 & 18.59 & 10 & 1 & 0 & 0 & 1 & 0.5 \\
\hline 51 & M / 28 & $S / X$ & 10 & $14.20 \pm 0.73$ & 14.26 & 0.04 & 12.32 & 14.91 & 0 & 0 & 0 & 0 & 0 & 0 \\
\hline 52 & M / 28 & $S / X$ & 10 & $13.81 \pm 0.84$ & 13.94 & 0.05 & 11.67 & 14.91 & 0 & 1 & 1 & 0 & 2 & 1.0 \\
\hline 53 & $\mathrm{M} / 28$ & NS / X & 3 & $14.63 \pm 1.78$ & 14.10 & 0.22 & 11.54 & 19.87 & 11 & 2 & 0 & 0 & 2 & 1.0 \\
\hline 54 & F / 28 & NS / X & 4 & $15.18 \pm 1.72$ & 14.74 & 0.19 & 11.54 & 19.23 & 15 & 2 & 0 & 0 & 2 & 1.0 \\
\hline 55 & M / 28 & $S / X$ & 4 & $14.79 \pm 1.87$ & 14.74 & 0.24 & 10.90 & 19.23 & 12 & 0 & 0 & 0 & 0 & 0 \\
\hline 56 & F / 29 & S & 2 & $15.42 \pm 1.32$ & 15.38 & 0.11 & 12.18 & 18.59 & 12 & 1 & 0 & 0 & 1 & 0.5 \\
\hline 57 & M / 29 & $S / X$ & 3 & $15.18 \pm 2.02$ & 15.38 & 0.27 & 11.54 & 19.87 & 20 & 1 & 1 & 1 & 3 & 1.5 \\
\hline 58 & F / 29 & NS & 5 & $12.97 \pm 0.66$ & 12.97 & 0.03 & 11.02 & 13.61 & 0 & 1 & 0 & 0 & 1 & 0.5 \\
\hline 59 & M / 29 & NS / X & 2 & $14.23 \pm 1.29$ & 14.10 & 0.12 & 10.90 & 17.31 & 1 & 0 & 0 & 1 & 1 & 0.5 \\
\hline 60 & F / 29 & NS & 7 & $13.58 \pm 1.54$ & 13.46 & 0.18 & 9.62 & 16.67 & 0 & 2 & 0 & 0 & 2 & 1.0 \\
\hline 61 & F / 29 & NS & 7 & $13.65 \pm 1.55$ & 13.46 & 0.18 & 10.26 & 17.31 & 2 & 0 & 0 & 0 & 0 & 0 \\
\hline 62 & M / 29 & $S / x$ & 4 & $14.41 \pm 1.63$ & 14.74 & 0.19 & 10.90 & 19.23 & 7 & 1 & 0 & 0 & 1 & 0.5 \\
\hline 63 & $\mathrm{~F} / 30$ & S & 4 & $14.55 \pm 1.53$ & 14.10 & 0.16 & 10.90 & 19.23 & 6 & 0 & 0 & 0 & 0 & 0 \\
\hline 64 & $\mathrm{M} / 30$ & S & 4 & $14.26 \pm 1.43$ & 14.10 & 0.14 & 10.26 & 17.95 & 4 & 0 & 0 & 0 & 0 & 0 \\
\hline 65 & M / 30 & NS & 10 & $14.03 \pm 0.71$ & 14.26 & 0.04 & 12.32 & 14.91 & 0 & 0 & 0 & 0 & 0 & 0 \\
\hline 66 & $\mathrm{~F} / 30$ & NS & 1 & $14.42 \pm 1.10$ & 14.26 & 0.08 & 12.32 & 17.50 & 4 & 0 & 0 & 0 & 0 & 0 \\
\hline 67 & $\mathrm{~F} / 30$ & NS & 3 & $13.92 \pm 0.89$ & 14.26 & 0.06 & 12.32 & 15.56 & 0 & 1 & 0 & 0 & 1 & 0.5 \\
\hline 68 & $\mathrm{M} / 30$ & NS / X & 2 & $14.00 \pm 1.42$ & 14.10 & 0.14 & 10.91 & 17.31 & 3 & 1 & 0 & 0 & 1 & 0.5 \\
\hline 69 & M / 31 & S & 3 & $14.89 \pm 1.48$ & 14.74 & 0.15 & 11.54 & 19.87 & 6 & 0 & 0 & 0 & 0 & 0 \\
\hline 70 & F / 31 & S & 4 & $13.98 \pm 1.31$ & 14.10 & 0.12 & 10.26 & 17.31 & 1 & 2 & 0 & 0 & 2 & 1.0 \\
\hline 71 & M / 31 & $S$ & 2 & $14.29 \pm 1.56$ & 14.10 & 0.17 & 11.54 & 17.95 & 4 & 0 & 0 & 1 & 1 & 0.5 \\
\hline 72 & $\mathrm{~F} / 31$ & S & 7 & $13.51 \pm 1.47$ & 13.46 & 0.16 & 10.26 & 16.67 & 0 & 1 & 0 & 0 & 1 & 0.5 \\
\hline 73 & $\mathrm{~F} / 32$ & $S / X$ & 3 & $15.22 \pm 1.80$ & 15.38 & 0.21 & 10.90 & 19.23 & 20 & 0 & 0 & 0 & 0 & 0 \\
\hline 74 & $\mathrm{~F} / 32$ & NS & 5 & $12.62 \pm 0.98$ & 12.97 & 0.08 & 9.08 & 14.26 & 0 & 0 & 0 & 0 & 0 & 0 \\
\hline 75 & M / 32 & NS & 3 & $13.83 \pm 1.35$ & 13.46 & 0.13 & 10.90 & 17.31 & 1 & 0 & 0 & 1 & 1 & 0.5 \\
\hline 76 & $\mathrm{M} / 32$ & NS & 3 & $13.08 \pm 1.48$ & 12.82 & 0.17 & 9.62 & 17.31 & 2 & 0 & 0 & 0 & 0 & 0 \\
\hline 77 & M / 32 & $S / X$ & 2 & $15.15 \pm 1.26$ & 15.06 & 0.11 & 12.18 & 17.31 & 6 & 2 & 0 & 0 & 2 & 1.0 \\
\hline 78 & F / 32 & S & 7 & $14.30 \pm 1.30$ & 14.10 & 0.11 & 10.90 & 17.31 & 1 & 1 & 0 & 1 & 2 & 1.0 \\
\hline 79 & M / 32 & S & 10 & $14.05 \pm 1.35$ & 14.26 & 0.13 & 11.02 & 16.86 & 2 & 2 & 0 & 0 & 2 & 1.0 \\
\hline 80 & $\mathrm{M} / 32$ & $S / X$ & 4 & $14.97 \pm 1.80$ & 14.74 & 0.22 & 11.54 & 18.59 & 18 & 0 & 1 & 0 & 1 & 0.5 \\
\hline 81 & M / 33 & NS / X & 2 & $14.94 \pm 1.52$ & 14.74 & 0.15 & 10.90 & 18.59 & 9 & 2 & 0 & 0 & 2 & 1.0 \\
\hline 82 & $\mathrm{M} / 33$ & NS & 5 & $12.76 \pm 0.86$ & 12.97 & 0.06 & 9.72 & 13.61 & 0 & 1 & 0 & 0 & 1 & 0.5 \\
\hline 83 & $\mathrm{~F} / 33$ & NS & 1 & $13.95 \pm 1.03$ & 13.94 & 0.08 & 11.67 & 16.86 & 2 & 0 & 0 & 0 & 0 & 0 \\
\hline 84 & $\mathrm{M} / 33$ & $S / X$ & 3 & $13.17 \pm 1.60$ & 12.82 & 0.19 & 9.62 & 16.67 & 0 & 1 & 0 & 0 & 1 & 0.5 \\
\hline 85 & $\mathrm{M} / 33$ & $S / X$ & 2 & $14.60 \pm 1.62$ & 14.74 & 0.18 & 10.26 & 17.95 & 10 & 1 & 1 & 0 & 2 & 1.0 \\
\hline 86 & F / 33 & S & 6 & $14.72 \pm 1.22$ & 14.74 & 0.10 & 11.54 & 16.67 & 0 & 1 & 0 & 0 & 1 & 0.5 \\
\hline 87 & M / 34 & $S / x$ & 2 & $14.80 \pm 1.54$ & 14.74 & 0.16 & 11.54 & 18.59 & 9 & 0 & 1 & 0 & 1 & 0.5 \\
\hline 88 & M / 35 & NS / $\mathrm{X}$ & 2 & $14.41 \pm 1.59$ & 14.42 & 0.18 & 10.90 & 17.31 & 3 & 0 & 0 & 1 & 1 & 0.5 \\
\hline 89 & $\mathrm{~F} / 35$ & NS & 3 & $13.99 \pm 1.51$ & 14.10 & 0.16 & 10.26 & 16.67 & 0 & 1 & 0 & 0 & 1 & 0.5 \\
\hline 90 & F / 35 & S & 4 & $14.60 \pm 1.35$ & 14.74 & 0.13 & 11.54 & 17.31 & 3 & 0 & 0 & 1 & 1 & 0.5 \\
\hline
\end{tabular}




\begin{tabular}{|c|c|c|c|c|c|c|c|c|c|c|c|c|c|c|}
\hline 91 & M / 35 & NS & 3 & $14.93 \pm 1.35$ & 14.74 & 0.12 & 12.18 & 18.59 & 7 & 0 & 0 & 0 & 0 & 0 \\
\hline 92 & M / 35 & NS & 3 & $13.13 \pm 1.34$ & 12.82 & 0.14 & 9.62 & 16.67 & 0 & 0 & 0 & 0 & 0 & 0 \\
\hline 93 & F / 35 & NS & 7 & $14.04 \pm 1.32$ & 14.10 & 0.12 & 10.26 & 17.31 & 1 & 1 & 0 & 0 & 1 & 0.5 \\
\hline 94 & $\mathrm{~F} / 35$ & NS & 12 & $13.95 \pm 1.63$ & 14.10 & 0.19 & 10.26 & 18.59 & 4 & 1 & 0 & 1 & 2 & 1.0 \\
\hline 95 & F / 35 & NS & 7 & $14.44 \pm 1.24$ & 14.74 & 0.11 & 10.90 & 17.31 & 1 & 1 & 0 & 0 & 1 & 0.5 \\
\hline 96 & M / 35 & NS / X & 4 & $14.89 \pm 1.62$ & 14.74 & 0.18 & 11.54 & 18.59 & 11 & 1 & 0 & 0 & 1 & 0.5 \\
\hline 97 & M / 36 & $S / X$ & 4 & $15.71 \pm 1.92$ & 16.03 & 0.23 & 10.90 & 19.23 & 26 & 0 & 0 & 0 & 0 & 0 \\
\hline 98 & $\mathrm{~F} / 36$ & S & 12 & $14.60 \pm 1.42$ & 14.42 & 0.14 & 10.90 & 17.31 & 4 & 1 & 0 & 0 & 1 & 0.5 \\
\hline 99 & M / 36 & NS / X & 2 & $14.63 \pm 1.73$ & 14.74 & 0.20 & 10.26 & 19.23 & 10 & 0 & 0 & 1 & 1 & 0.5 \\
\hline 100 & M / 36 & S & 4 & $14.72 \pm 1.54$ & 14.74 & 0.16 & 11.54 & 17.95 & 8 & 0 & 0 & 0 & 0 & 0 \\
\hline 101 & M / 36 & S & 3 & $15.27 \pm 1.41$ & 15.06 & 0.13 & 12.18 & 19.23 & 10 & 1 & 0 & 0 & 1 & 0.5 \\
\hline 102 & $\mathrm{~F} / 36$ & NS & 7 & $12.68 \pm 1.55$ & 12.82 & 0.19 & 9.62 & 16.67 & 0 & 0 & 0 & 1 & 1 & 0.5 \\
\hline 103 & M / 36 & NS / X & 9 & $15.47 \pm 1.44$ & 15.38 & 0.13 & 12.82 & 17.95 & 18 & 0 & 0 & 0 & 0 & 0 \\
\hline 104 & M / 37 & $S / x$ & 2 & $14.94 \pm 1.53$ & 14.74 & 0.16 & 11.54 & 18.59 & 12 & 2 & 0 & 0 & 2 & 1.0 \\
\hline 105 & $\mathrm{M} / 37$ & S & 3 & $14.70 \pm 1.45$ & 14.74 & 0.14 & 10.90 & 17.95 & 7 & 1 & 0 & 0 & 1 & 0.5 \\
\hline 106 & $\mathrm{~F} / 37$ & NS & 6 & $14.82 \pm 1.38$ & 14.74 & 0.13 & 12.18 & 17.95 & 3 & 0 & 1 & 0 & 1 & 0.5 \\
\hline 107 & $\mathrm{M} / 37$ & $S / X$ & 4 & $14.54 \pm 1.41$ & 14.74 & 0.14 & 10.26 & 17.95 & 5 & 1 & 0 & 1 & 2 & 1.0 \\
\hline 108 & $\mathrm{~F} / 38$ & NS & 2 & $12.10 \pm 1.49$ & 12.32 & 0.18 & 9.72 & 17.50 & 2 & 0 & 0 & 0 & 0 & 0 \\
\hline 109 & $\mathrm{M} / 38$ & $S / X$ & 10 & $14.28 \pm 0.67$ & 14.26 & 0.03 & 11.67 & 14.91 & 0 & 2 & 1 & 0 & 3 & 1.5 \\
\hline 110 & $\mathrm{M} / 38$ & S & 3 & $14.69 \pm 1.54$ & 14.74 & 0.16 & 10.90 & 17.95 & 9 & 1 & 0 & 0 & 1 & 0.5 \\
\hline 111 & $\mathrm{~F} / 38$ & NS & 4 & $14.88 \pm 1.39$ & 14.74 & 0.13 & 12.18 & 17.95 & 6 & 0 & 0 & 1 & 1 & 0.5 \\
\hline 112 & $\mathrm{M} / 38$ & S & 4 & $13.99 \pm 1.36$ & 14.10 & 0.13 & 10.90 & 17.95 & 1 & 0 & 0 & 1 & 1 & 0.5 \\
\hline 113 & $\mathrm{~F} / 38$ & NS / X & 4 & $14.20 \pm 1.77$ & 14.74 & 0.22 & 10.26 & 17.95 & 5 & 1 & 1 & 0 & 2 & 1.0 \\
\hline 114 & M / 39 & NS & 3 & $15.26 \pm 1.24$ & 15.38 & 0.10 & 12.18 & 17.31 & 6 & 1 & 0 & 0 & 1 & 0.5 \\
\hline 115 & M / 39 & NS & 3 & $12.73 \pm 1.58$ & 12.82 & 0.20 & 9.62 & 16.03 & 0 & 2 & 0 & 0 & 2 & 1.0 \\
\hline 116 & M / 39 & NS / AD & 3 & $15.39 \pm 1.41$ & 15.38 & 0.13 & 12.18 & 17.95 & 16 & 1 & 1 & 0 & 2 & 1.0 \\
\hline 117 & F / 39 & NS & 6 & $14.81 \pm 1.22$ & 14.74 & 0.10 & 12.18 & 17.95 & 1 & 0 & 0 & 0 & 0 & 0 \\
\hline 118 & $\mathrm{M} / 40$ & NS & 3 & $14.74 \pm 1.25$ & 14.74 & 0.11 & 12.18 & 17.31 & 2 & 1 & 0 & 1 & 2 & 1.0 \\
\hline 119 & $\mathrm{~F} / 41$ & NS & 4 & $14.72 \pm 1.41$ & 14.74 & 0.13 & 11.54 & 17.95 & 5 & 0 & 0 & 1 & 1 & 0.5 \\
\hline 120 & $\mathrm{M} / 41$ & S & 3 & $13.28 \pm 1.46$ & 13.46 & 0.16 & 10.26 & 17.31 & 1 & 0 & 0 & 0 & 0 & 0 \\
\hline 121 & $\mathrm{~F} / 41$ & NS & 4 & $15.04 \pm 1.45$ & 15.38 & 0.14 & 9.62 & 17.95 & 7 & 1 & 0 & 0 & 1 & 0.5 \\
\hline 122 & M / 41 & S / MRI & 3 & $15.17 \pm 1.66$ & 15.38 & 0.18 & 11.54 & 19.23 & 18 & 2 & 0 & 0 & 2 & 1.0 \\
\hline 123 & $\mathrm{~F} / 41$ & S & 6 & $14.81 \pm 1.27$ & 14.74 & 0.11 & 10.90 & 17.95 & 2 & 0 & 0 & 0 & 0 & 0 \\
\hline 124 & $\mathrm{~F} / 41$ & S & 7 & $14.31 \pm 1.42$ & 14.10 & 0.14 & 10.26 & 17.31 & 1 & 1 & 0 & 0 & 1 & 0.5 \\
\hline 125 & $\mathrm{M} / 42$ & NS / X & 3 & $15.55 \pm 1.65$ & 15.38 & 0.18 & 12.18 & 19.87 & 17 & 0 & 0 & 1 & 1 & 0.5 \\
\hline 126 & $\mathrm{~F} / 42$ & S & 4 & $14.15 \pm 1.21$ & 14.10 & 0.10 & 11.54 & 16.67 & 0 & 1 & 0 & 0 & 1 & 0.5 \\
\hline 127 & $\mathrm{M} / 42$ & NS / X & 2 & $14.04 \pm 1.42$ & 14.10 & 0.14 & 10.26 & 16.67 & 0 & 0 & 0 & 0 & 0 & 0 \\
\hline 128 & $\mathrm{M} / 42$ & S & 5 & $14.65 \pm 1.62$ & 14.74 & 0.18 & 10.90 & 17.95 & 1 & 0 & 0 & 0 & 0 & 0 \\
\hline 129 & M / 43 & S & 3 & $13.09 \pm 1.39$ & 12.82 & 0.15 & 10.26 & 17.31 & 1 & 1 & 0 & 0 & 1 & 0.5 \\
\hline 130 & $\mathrm{~F} / 43$ & S & 6 & $14.67 \pm 1.46$ & 14.74 & 0.15 & 10.90 & 17.95 & 4 & 1 & 0 & 0 & 1 & 0.5 \\
\hline 131 & $\mathrm{~F} / 44$ & S & 4 & $14.72 \pm 1.64$ & 14.74 & 0.18 & 10.90 & 19.87 & 8 & 0 & 0 & 1 & 1 & 0.5 \\
\hline 132 & M / 44 & NS & 9 & $12.67 \pm 1.25$ & 12.64 & 0.12 & 10.37 & 14.91 & 0 & 1 & 0 & 0 & 1 & 0.5 \\
\hline 133 & M / 44 & S & 4 & $14.90 \pm 1.72$ & 14.74 & 0.20 & 10.26 & 21.15 & 13 & 0 & 1 & 1 & 2 & 1.0 \\
\hline 134 & $\mathrm{~F} / 45$ & S & 4 & $13.37 \pm 1.19$ & 13.46 & 0.11 & 10.26 & 17.31 & 1 & 1 & 0 & 0 & 1 & 0.5 \\
\hline 135 & $\mathrm{~F} / 45$ & NS & 10 & $14.54 \pm 1.65$ & 14.74 & 0.19 & 10.26 & 17.31 & 7 & 1 & 0 & 1 & 2 & 1.0 \\
\hline 136 & $\mathrm{~F} / 45$ & NS & 6 & $15.04 \pm 1.40$ & 15.38 & 0.13 & 11.54 & 17.95 & 6 & 1 & 0 & 0 & 1 & 0.5 \\
\hline 137 & $\mathrm{~F} / 45$ & S & 7 & $14.09 \pm 1.39$ & 14.10 & 0.14 & 10.26 & 17.31 & 1 & 0 & 0 & 0 & 0 & 0 \\
\hline 138 & F / 45 & NS & 7 & $14.22 \pm 1.29$ & 14.10 & 0.12 & 10.90 & 17.95 & 1 & 0 & 0 & 1 & 1 & 0.5 \\
\hline
\end{tabular}




\begin{tabular}{|c|c|c|c|c|c|c|c|c|c|c|c|c|c|c|}
\hline 139 & $\mathrm{M} / 45$ & S & 10 & $14.88 \pm 1.72$ & 14.74 & 0.20 & 10.26 & 19.87 & 9 & 4 & 0 & 1 & 5 & 2.5 \\
\hline 140 & F / 46 & S & 4 & $14.06 \pm 1.51$ & 14.10 & 0.16 & 10.26 & 17.31 & 1 & 1 & 1 & 0 & 2 & 1.0 \\
\hline 141 & $\mathrm{M} / 46$ & S & 3 & $14.24 \pm 1.36$ & 14.10 & 0.13 & 11.54 & 17.95 & 5 & 2 & 0 & 0 & 2 & 1.0 \\
\hline 142 & $\mathrm{M} / 46$ & $S / x$ & 10 & $14.57 \pm 0.74$ & 14.91 & 0.04 & 11.67 & 15.56 & 0 & 2 & 0 & 1 & 3 & 1.5 \\
\hline 143 & $\mathrm{~F} / 47$ & S & 4 & $13.94 \pm 1.38$ & 14.10 & 0.14 & 10.90 & 16.67 & 0 & 2 & 0 & 0 & 2 & 1.0 \\
\hline 144 & $\mathrm{M} / 47$ & S / MRI & 4 & $15.08 \pm 1.51$ & 14.74 & 0.15 & 11.54 & 18.59 & 11 & 1 & 0 & 0 & 1 & 0.5 \\
\hline 145 & $\mathrm{~F} / 47$ & NS & 7 & $12.83 \pm 1.32$ & 12.82 & 0.14 & 9.62 & 16.03 & 0 & 1 & 0 & 0 & 1 & 0.5 \\
\hline 146 & $\mathrm{~F} / 47$ & S & 6 & $14.73 \pm 1.39$ & 14.74 & 0.13 & 10.90 & 17.31 & 2 & 0 & 0 & 1 & 1 & 0.5 \\
\hline 147 & $\mathrm{~F} / 48$ & $S$ & 4 & $13.81 \pm 1.41$ & 13.78 & 0.14 & 10.90 & 16.67 & 0 & 1 & 0 & 1 & 2 & 1.0 \\
\hline 148 & $\mathrm{~F} / 49$ & NS & 5 & $14.07 \pm 1.54$ & 14.10 & 0.17 & 10.90 & 16.67 & 0 & 0 & 0 & 0 & 0 & 0 \\
\hline 149 & $\mathrm{~F} / 49$ & NS & 3 & $13.94 \pm 1.33$ & 14.74 & 0.13 & 10.90 & 17.31 & 2 & 0 & 0 & 0 & 0 & 0 \\
\hline 150 & F / 49 & NS & 4 & $14.41 \pm 1.36$ & 14.74 & 0.13 & 11.54 & 17.95 & 4 & 1 & 0 & 0 & 1 & 0.5 \\
\hline 151 & F / 49 & S & 7 & $13.81 \pm 1.41$ & 13.78 & 0.14 & 10.90 & 16.67 & 0 & 0 & 0 & 0 & 0 & 0 \\
\hline 152 & $\mathrm{M} / 50$ & NS & 5 & $12.52 \pm 0.91$ & 12.32 & 0.07 & 9.72 & 13.61 & 0 & 1 & 0 & 0 & 1 & 0.5 \\
\hline 153 & $\mathrm{~F} / 50$ & NS & 10 & $14.86 \pm 1.52$ & 14.74 & 0.15 & 10.26 & 17.95 & 9 & 2 & 0 & 0 & 2 & 1.0 \\
\hline 154 & $\mathrm{~F} / 50$ & NS & 6 & $14.76 \pm 1.37$ & 14.74 & 0.13 & 10.90 & 17.95 & 5 & 1 & 0 & 0 & 1 & 0.5 \\
\hline 155 & $\mathrm{~F} / 51$ & NS & 4 & $14.62 \pm 1.50$ & 14.74 & 0.15 & 11.54 & 18.59 & 8 & 1 & 0 & 0 & 1 & 0.5 \\
\hline 156 & $\mathrm{~F} / 51$ & S & 4 & $14.04 \pm 1.39$ & 14.10 & 0.14 & 10.90 & 17.95 & 2 & 1 & 0 & 1 & 2 & 1.0 \\
\hline 157 & M / 51 & NS & 5 & $14.00 \pm 1.47$ & 14.10 & 0.15 & 10.90 & 16.67 & 0 & 0 & 0 & 0 & 0 & 0 \\
\hline 158 & $\mathrm{~F} / 51$ & NS & 6 & $14.15 \pm 1.44$ & 14.10 & 0.15 & 10.90 & 17.31 & 1 & 1 & 1 & 0 & 2 & 1.0 \\
\hline 159 & $\mathrm{~F} / 51$ & NS & 7 & $14.02 \pm 1.57$ & 14.10 & 0.18 & 10.26 & 17.95 & 1 & 1 & 0 & 0 & 1 & 0.5 \\
\hline 160 & $\mathrm{~F} / 52$ & NS & 6 & $13.22 \pm 1.40$ & 13.14 & 0.15 & 10.26 & 16.67 & 0 & 0 & 0 & 0 & 0 & 0 \\
\hline 161 & $\mathrm{~F} / 52$ & $\mathrm{NS} / \mathrm{AD}$ & 12 & $15.06 \pm 1.42$ & 15.38 & 0.13 & 12.18 & 17.95 & 9 & 0 & 0 & 0 & 0 & 0 \\
\hline 162 & $\mathrm{~F} / 53$ & NS & 6 & $13.93 \pm 1.59$ & 14.10 & 0.18 & 9.62 & 16.67 & 0 & 1 & 0 & 0 & 1 & 0.5 \\
\hline 163 & $\mathrm{~F} / 53$ & NS & 6 & $13.86 \pm 1.43$ & 13.78 & 0.15 & 10.90 & 17.31 & 2 & 0 & 0 & 0 & 0 & 0 \\
\hline 164 & $\mathrm{~F} / 53$ & NS & 6 & $15.10 \pm 1.43$ & 15.38 & 0.14 & 11.54 & 17.95 & 8 & 1 & 0 & 0 & 1 & 0.5 \\
\hline 165 & $\mathrm{~F} / 54$ & S & 4 & $14.17 \pm 1.31$ & 14.10 & 0.12 & 11.54 & 17.31 & 1 & 1 & 0 & 0 & 1 & 0.5 \\
\hline 166 & $\mathrm{~F} / 56$ & S & 6 & $13.61 \pm 1.54$ & 13.46 & 0.17 & 10.26 & 17.31 & 1 & 1 & 0 & 0 & 1 & 0.5 \\
\hline 167 & $\mathrm{M} / 58$ & NS & 5 & $13.81 \pm 1.51$ & 14.10 & 0.17 & 10.90 & 17.31 & 2 & 1 & 0 & 0 & 1 & 0.5 \\
\hline 168 & F / 59 & NS & 4 & $13.76 \pm 1.16$ & 14.10 & 0.10 & 10.90 & 17.31 & 1 & 0 & 0 & 0 & 0 & 0 \\
\hline 169 & $\mathrm{~F} / 61$ & NS & 6 & $15.03 \pm 1.28$ & 14.74 & 0.11 & 12.18 & 17.31 & 1 & 1 & 0 & 0 & 1 & 0.5 \\
\hline 170 & F / 64 & NS & 6 & $14.35 \pm 1.39$ & 14.74 & 0.13 & 10.90 & 17.31 & 1 & 0 & 0 & 0 & 0 & 0 \\
\hline
\end{tabular}

F, female subject; M, male subject; NS, non-smoker; S-smoker; X, diagnostic exposure to X-rays; MRI, diagnostic exposure to magnetic resonance imaging; AD, antirheumatic drugs; Med., median; H, dispersion coefficient; LTN, long tailed nuclei; CA, chromosome aberrations $\mathbf{B}_{1}$, chromatid break; $\mathbf{B}_{2}$, chromosome break; Ac, acentric fragment.

ed in Table 1. Comparisons of group mean values are reported in Tables 2 and 3.

The results reported in Table 1 point to an inter-individual diversity among the subjects studied. As shown in Table 1, individual values of DNA migration in white blood cells were in the range 12.10 \pm 1.49 to $15.91 \pm 2.24$. Average value of DNA migration in the whole group studied was $14.25 \pm 0.80$ $\mu \mathrm{m}$ and the median was $14.31 \mu \mathrm{m}$ (Table 1). The number of long-tailed nuclei (LTN), e.g. comets with tail length exceeding the 95th percentile for the considered parameter, is also reported. Cells with tail length values below the cut-off $(16.67 \mu \mathrm{m})$ were classified as "undamaged", and those with higher values as "damaged". Scoring 100 comets per subject, 5 LTN are expected on average, and 8 LTN per sub- ject with a probability of $5 \%$. As reported in Table 1 , the distribution of "damaged" cells was clearly wider in subjects exposed to diagnostic X-rays and smokers compared to other subjects. The average number of LTN in the whole group was 4.75 (range: $0-35)$. Altogether 128 subjects $(75 \%)$ had no or less than 8 LTN per 100 comets scored, while other 42 subjects $(25 \%)$ having 8 or more LTN in 100 comets were considered as "outliers". The distribution of the "outliers" with respect to their smoking status or medicinal exposure is shown on Fig. 1. It has to be pointed out that among non-smokers without any medical exposure only four subjects were "outliers" and they usually had 8 or 9 LTN per 100 comets (Table 1). 


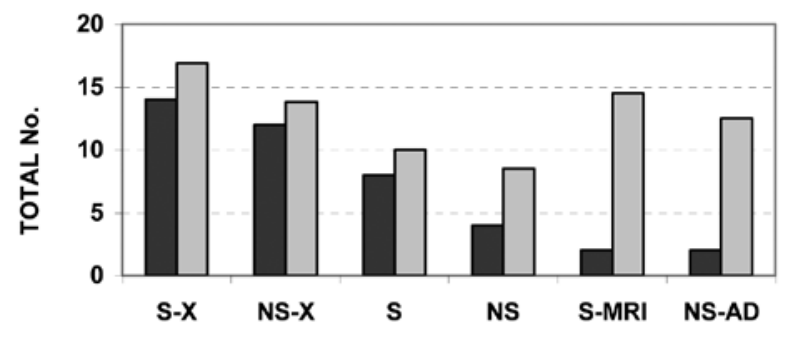

口SUBJECTS WITH LTN 口 LTN PER SUBJECT

Figure 1. The distribution of "outliers" (i.e. subjects with 8 and more long tailed nuclei - LTN per 100 comets) with respect to their smoking status or medical exposure.

NS, non-smoker; S, smoker; $X$, diagnostic exposure to $X$ rays; MRI, diagnostic exposure to magnetic resonance imaging; $\mathrm{AD}$, antirheumatic drugs.

Individual differences in the level of primary DNA damage could be also seen when analyzing the values of dispersion coefficient $(\mathrm{H})$. High value of $\mathrm{H}$ indicates an increase in the proportion of cells with a high extent of damage (Table 1).

When multifactor ANOVA on logarithmically transformed data was applied for tail lengths, no statistically significant differences between individuals as related to their age were found out. Male subjects had a little higher, but not statistically significant, mean value of comet tail length compared to female subjects. However, their average number of LTN (5.89 LTN per 100 comets) was significantly increased compared to female subjects (3.33 LTN per 100 comets) (Table 2).

Smoking habit significantly influenced the levels of primary DNA damage. Smokers had an average tail length of $14.49 \pm 0.63 \mu \mathrm{m}$ and $5.95 \mathrm{LTN}$ per 100 comets. Both parameters were significantly increased with respect to non-smokers (average tail length of $14.05 \pm 0.88 \mu \mathrm{m}$ and $3.78 \mathrm{LTN}$ per 100 comets) (Table 2).

Although only two subjects reported diagnostic exposure to MRI, or occasional intake of antirheumatic drugs, the levels of primary DNA

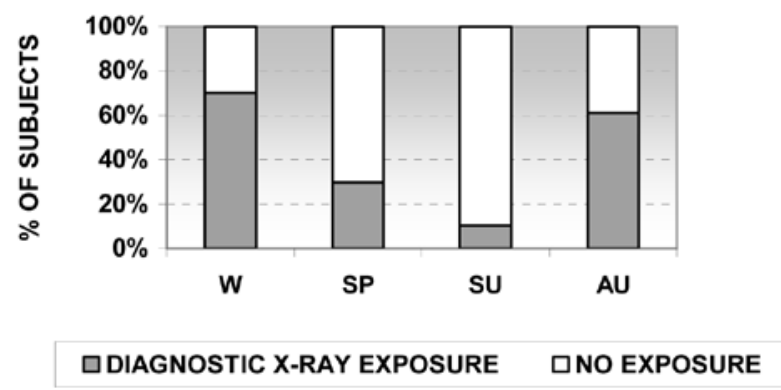

Figure 2. The distribution of subjects with diagnostic $X$-ray exposure dependent on the season of blood sampling.

W, winter; SP, spring; SU, summer; AU, autumn.

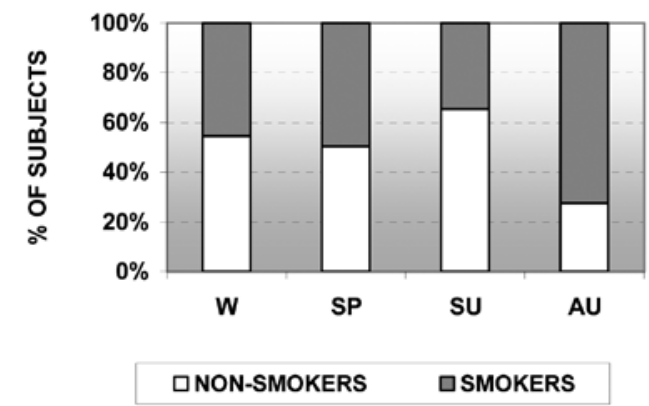

Figure 3. The distribution of smoking and non-smoking subjects with respect to the season of blood sampling.

W, winter; SP, spring; SU, summer; AU, autumn.

damage in their white blood cells were also significantly increased (Table 2). Diagnostic X-ray exposure most efficiently enhanced the levels of primary DNA damage: the mean tail length in 52 subjects with medical exposure was $14.69 \pm 0.60$ $\mu \mathrm{m}$, while $8.92 \mathrm{LTN}$ per 100 comets were recorded (Table 2).

Although significant differences between mean tail lengths and the number of LTN recorded in blood samples collected in winter and summer were observed, statistical analysis confirmed that they were mostly influenced by diagnostic X-ray exposure and smoking habits. As shown on Fig. 2, the most of blood samples of subjects with diagnostic X-ray exposure were collected in winter, while the most of blood samples collected in summer were donated by non-smoking subjects (Fig. 3).

These observations prompted us to separately analyse the subpopulation without any diagnostic exposure $(n=114)$ with idea to evaluate the effects of gender, age, smoking, and season of blood sampling on the level of primary DNA damage in their white blood cells. The results of this evaluation are reported in Table 3. They confirmed that smoking significantly influenced the levels of primary DNA

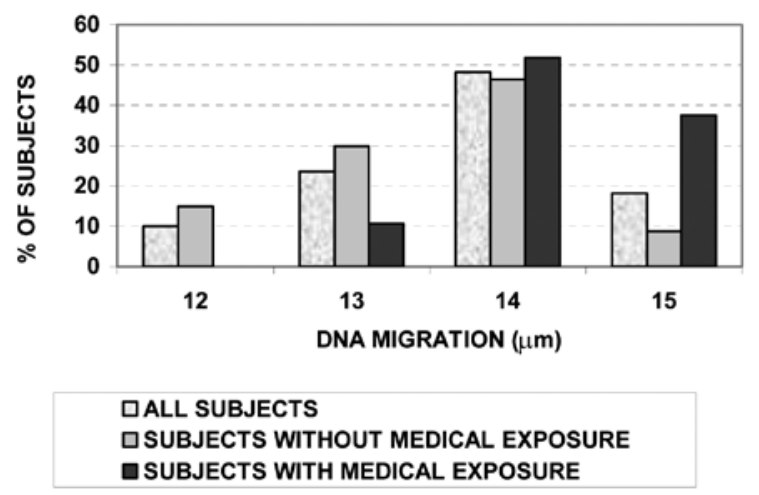

Figure 4. The distribution of the mean values of DNA migration recorded in white blood cells of 170 healthy blood donors.

The values obtained for the whole study group vs. subgroups with or without medical exposure are compared. 
Table 2. Results of alkaline comet assay and analysis of structural chromosome aberrations (CA) in white blood cells of $\mathbf{1 7 0}$ healthy blood donors, expressed as group mean values.

DNA migration was evaluated by measuring 100 comets per subject, while incidence of CA was evaluated by analysing 200 metaphases per subject.

\begin{tabular}{|c|c|c|c|c|c|c|c|c|}
\hline \multirow{3}{*}{ Subgroups } & \multirow{3}{*}{$\begin{array}{l}\text { No. of } \\
\text { subjects }\end{array}$} & \multirow{3}{*}{$\begin{array}{l}\text { DNA migration }(\mu \mathrm{m}) \\
\text { Mean } \pm \text { S.D. (median) } \\
\text { Range }\end{array}$} & \multirow{3}{*}{$\begin{array}{l}\text { No. of } \\
\underline{\text { LTN }} \\
\text { Range }\end{array}$} & \multirow{2}{*}{\multicolumn{4}{|c|}{$\begin{array}{l}\text { Structural CA } \\
\text { (Mean/Range) }\end{array}$}} & \multirow{3}{*}{$\begin{array}{l}\% \text { of cells } \\
\text { with CA }\end{array}$} \\
\hline & & & & & & & & \\
\hline & & & & $\mathrm{B}_{1}$ & $\mathrm{~B}_{2}$ & Ac & $\Sigma$ & \\
\hline \multicolumn{9}{|l|}{ SEX } \\
\hline \multirow{2}{*}{ Female } & \multirow{2}{*}{76} & $14.16 \pm 0.75(14.15)$ & 3.33 & 0.62 & 0.05 & 0.18 & 0.86 & 0.43 \\
\hline & & $12.10-15.91$ & $0-35$ & $0-2$ & $0-1$ & $0-1$ & $0-2$ & $0-1$ \\
\hline \multirow{2}{*}{ Male } & \multirow{2}{*}{94} & $14.32 \pm 0.85(14.51)$ & $5.89^{\mathrm{a}}$ & 0.72 & 0.15 & 0.18 & 1.05 & 0.53 \\
\hline & & $12.43-15.71$ & $0-26$ & $0-4$ & $0-2$ & $0-5$ & $0-3$ & $0-2.5$ \\
\hline \multicolumn{9}{|c|}{ SMOKING HABITS } \\
\hline \multirow{2}{*}{ Non-smokers } & \multirow{2}{*}{94} & $14.05 \pm 0.88(14.06)$ & 3.78 & 0.57 & 0.09 & 0.16 & 0.82 & 0.41 \\
\hline & & $12.10-15.55$ & $0-21$ & $0-2$ & $0-2$ & $0-1$ & $0-2$ & $0-1$ \\
\hline \multirow{2}{*}{ NS-F } & \multirow{2}{*}{47} & $14.05 \pm 0.78(14.04)$ & 2.79 & 0.62 & 0.06 & 0.13 & 0.81 & 0.40 \\
\hline & & $12.10-15.18$ & $0-15$ & $0-2$ & $0-1$ & $0-1$ & $0-2$ & $0-1$ \\
\hline \multirow{2}{*}{ NS-M } & \multirow{2}{*}{47} & $14.06 \pm 0.97(14.15)$ & 4.77 & 0.53 & 0.11 & 0.19 & 0.83 & 0.41 \\
\hline & & $12.43-15.55$ & $0-21$ & $0-2$ & $0-2$ & $0-1$ & $0-2$ & $0-1$ \\
\hline \multirow{2}{*}{ Smokers } & \multirow{2}{*}{76} & $14.49 \pm 0.63^{\mathbf{b}}(14.56)$ & $5.95^{b}$ & 0.80 & 0.13 & 0.21 & $1.14^{\mathrm{b}}$ & $0.57^{\mathbf{b}}$ \\
\hline & & $13.09-15.91$ & $0-35$ & $0-4$ & $0-2$ & $0-1$ & 0.5 & $0-2.5$ \\
\hline S-F & 29 & $14.36 \pm 0.65(14.30)$ & 4.21 & 0.62 & 0.03 & 0.28 & 0.93 & 0.47 \\
\hline $\mathrm{S}-\mathrm{F}$ & 29 & $13.31-15.91$ & $0-35$ & $0-2$ & $0-1$ & $0-1$ & $0-2$ & $0-1$ \\
\hline S-M & 47 & $14.58 \pm 0.61(14.65)$ & 7.02 & 0.91 & 0.19 & 0.17 & 1.28 & 0.64 \\
\hline S-lVI & $4 /$ & 13.09-15.71 & $0-26$ & $0-4$ & $0-2$ & $0-1$ & $0-5$ & $0-2.5$ \\
\hline AGE (years) & & & & & & & & \\
\hline & & $14.20 \pm 0.92(14.23)$ & 5.45 & 0.63 & 0.13 & 0.13 & 0.89 & 0.44 \\
\hline $20-29$ & 62 & $12.43-15.91$ & $0-35$ & $0-2$ & $0-2$ & $0-1$ & $0-3$ & $0-1.5$ \\
\hline & & $14.31 \pm 0.79(14.44)$ & 5.22 & 0.64 & 0.13 & 0.20 & 0.96 & 0.48 \\
\hline $30-39$ & 55 & $12.10-15.71$ & $0-26$ & $0-2$ & $0-1$ & $0-1$ & $0-3$ & $0-1.5$ \\
\hline $40-49$ & & $14.30 \pm 0.68(14.36)$ & 3.82 & 0.79 & 0.06 & 0.32 & 1.18 & 0.59 \\
\hline $40-49$ & 34 & $12.67-15.55$ & $0-18$ & $0-4$ & $0-1$ & $0-1$ & $0-5$ & $0-2.5$ \\
\hline $50-64$ & & $14.15 \pm 0.66(14.04)$ & 2.74 & 0.74 & 0.05 & 0.05 & 0.84 & 0.42 \\
\hline $50-64$ & 19 & $12.52-15.10$ & $0-9$ & $0-2$ & $0-1$ & $0-1$ & $0-2$ & $0-1$ \\
\hline TIME OF BLOOD & ING & & & & & & & \\
\hline & & $14.49 \pm 0.82^{\mathrm{c}}(14.63)$ & $7.40^{\mathrm{d}}$ & 0.68 & 0.11 & 0.16 & $0.95^{\mathrm{c}}$ & $0.48^{\mathrm{c}}$ \\
\hline Winter (1-III) & 62 & $12.10-15.91$ & $0-35$ & $0-2$ & $0-2$ & $0-1$ & $0-3$ & $0-1.5$ \\
\hline Spring (IV-VI) & 63 & $14.17 \pm 0.81(14.35)$ & 3.94 & 0.59 & 0.10 & 0.16 & 0.84 & 0.42 \\
\hline spring (IV-vI) & 00 & $12.43-15.71$ & $0-26$ & $0-2$ & $0-1$ & $0-1$ & $0-2$ & $0-1$ \\
\hline Summer (VII-IX) & 20 & $13.66 \pm 0.76(13.73)$ & 1.45 & 0.55 & _- & 0.20 & 0.75 & 0.38 \\
\hline sumtries (vil-i人) & 20 & $12.54-15.47$ & $0-18$ & $0-2$ & - & $0-1$ & $0-2$ & $0-1$ \\
\hline Autumn (X-XII) & 25 & $14.32 \pm 0.47(14.20)$ & 2.84 & 1.00 & 0.20 & 0.28 & $1.48^{\mathrm{d}}$ & $0.74^{\mathrm{d}}$ \\
\hline Autumn $(\lambda-\lambda I I)$ & 25 & $13.56-15.06$ & $0-9$ & $0-4$ & $0-2$ & $0-1$ & $0-5$ & $0-2.5$ \\
\hline MEDICINAL EXI & & & & & & & & \\
\hline None & 114 & $14.01 \pm 0.79(14.05)$ & 2.54 & 0.63 & 0.05 & 0.18 & 0.86 & 0.43 \\
\hline None & 114 & $12.10-15.55$ & $0-13$ & $0-4$ & $0-2$ & $0-1$ & $0-5$ & $0-2.5$ \\
\hline X-Ravs & 52 & $14.69 \pm 0.60^{\mathrm{e}}(15.20)$ & $8.92^{\mathbf{e}}$ & 0.75 & $0.21^{\mathrm{e}}$ & 0.21 & 1.17 & 0.59 \\
\hline$\lambda-$ kays & 52 & $13.17-15.91$ & $0-35$ & $0-2$ & $0-2$ & $0-1$ & $0-3$ & $0-1.5$ \\
\hline & & $15.23 \pm 0.23^{\mathrm{e}}(15.23)$ & $12.50^{\mathrm{e}}$ & 0.5 & $0.5^{\mathrm{e}}$ & & 1.00 & 0.50 \\
\hline MRI & 2 & $15.06-15.23$ & $9-16$ & $0-1$ & $0-1$ & - & $0-2$ & $0-1$ \\
\hline & & $15.13 \pm 0.06^{\mathrm{e}}(15.13)$ & $14.50^{\mathrm{e}}$ & 1.5 & & & $1.50^{\mathrm{e}}$ & $0.75^{\mathrm{e}}$ \\
\hline $\mathrm{AD}$ & 2 & $15.07-15.17$ & $11-18$ & $1-2$ & - & - & $1-2$ & $0.5-1$ \\
\hline All subiects & 170 & $14.25 \pm 0.80(14.31)$ & 4.75 & 0.68 & 0.11 & 0.18 & 0.96 & 0.48 \\
\hline All suidjects & $1 / 0$ & $12.10-15.91$ & 0-35 & $0-4$ & $0-2$ & $0-1$ & $0-5$ & $0-2.5$ \\
\hline
\end{tabular}

F, female subject; $\mathrm{M}$, male subject; NS, non-smoker; S, smoker; $\mathrm{X}$, diagnostic exposure to $\mathrm{X}$-rays; MRI, diagnostic exposure to magnetic resonance imaging; $\mathrm{AD}$, antirheumatic drugs; $\mathrm{LTN}$, long tailed nuclei; $\mathrm{CA}$, chromosome aberrations; $\mathrm{B}_{1}$, chromatid break; $\mathrm{B}_{2}$, chromosome break; Ac, acentric fragment. Multiple comparisons were made using multifactor ANOVA with post-hoc Scheffé test; significantly increased values $(P<0.05)$ were: ${ }^{a}$ with regard to female subjects; ${ }^{b}$ with regard to non-smokers; ${ }^{c}$ with regard to samples collected in summer; ${ }^{d}$ with regard to samples collected in all other seasons; ${ }^{e}$ with regard to subjects without any medical exposure.

damage, while gender and age did not significantly contribute to the pattern of DNA migration in white blood cells. Although the highest levels of primary
DNA damage were recorded in blood samples collected in autumn, they were mostly influenced by smoking status. 
Table 3. Results of alkaline comet assay and analysis of structural chromosome aberrations in white blood cells of 114 healthy blood donors, without any medicinal exposure, expressed as group mean values.

DNA migration was evaluated by measuring 100 comets per subject, while incidence of CA was evaluated by analysing 200 metaphases per subject.

\begin{tabular}{|c|c|c|c|c|c|c|c|c|}
\hline \multirow{3}{*}{ Subgroups } & \multirow{3}{*}{$\begin{array}{l}\text { No. of } \\
\text { subjects }\end{array}$} & \multirow{3}{*}{$\begin{array}{l}\text { DNA migration }(\mu \mathrm{m}) \\
\text { Mean } \pm \text { S.D. (median) } \\
\text { Range }\end{array}$} & \multirow{3}{*}{$\begin{array}{l}\text { No. of } \\
\text { LTN } \\
\text { Range }\end{array}$} & \multirow{2}{*}{\multicolumn{4}{|c|}{$\begin{array}{l}\text { Structural CA } \\
\text { (Mean/Range) }\end{array}$}} & \multirow{3}{*}{$\begin{array}{l}\text { Structural } \\
\text { CA } \\
\text { (Mean / } \\
\text { Range) }\end{array}$} \\
\hline & & & & & & & & \\
\hline & & & & $\mathrm{B}_{1}$ & $\mathrm{~B}_{2}$ & Ac & $\Sigma$ & \\
\hline \multicolumn{9}{|l|}{ SEX } \\
\hline \multirow{2}{*}{ Female } & \multirow{2}{*}{69} & $14.09 \pm 0.71(14.07)$ & 2.25 & 0.64 & 0.04 & 0.19 & 0.87 & 0.43 \\
\hline & & $12.10-15.42$ & $0-12$ & $0-2$ & $0-1$ & $0-1$ & $0-2$ & $0-1$ \\
\hline \multirow{2}{*}{ Male } & \multirow{2}{*}{45} & $13.90 \pm 0.91(14.03)$ & 2.98 & 0.62 & 0.07 & 0.16 & 0.84 & 0.42 \\
\hline & & $12.43-15.55$ & $0-13$ & $0-4$ & $0-2$ & $0-1$ & $0-5$ & $0-2.5$ \\
\hline \multicolumn{9}{|c|}{ SMOKING HABITS } \\
\hline \multirow{2}{*}{ Non-smokers } & \multirow{2}{*}{67} & $13.80 \pm 0.86(13.95)$ & 1.96 & 0.52 & 0.06 & 0.13 & 0.72 & 0.36 \\
\hline & & $12.10-15.26$ & $0-9$ & $0-2$ & $0-2$ & $0-1$ & $0-2$ & $0-1$ \\
\hline \multirow{2}{*}{ NS-F } & \multirow{2}{*}{47} & $14.00 \pm 0.78(14.02)$ & 2.33 & 0.60 & 0.05 & 0.14 & 0.79 & 0.40 \\
\hline & & $12.10-15.10$ & $0-9$ & $0-2$ & $0-1$ & $0-1$ & $0-2$ & $0-1$ \\
\hline \multirow{2}{*}{ NS-M } & \multirow{2}{*}{47} & $13.45 \pm 0.88(13.11)$ & 1.29 & 0.38 & 0.08 & 0.13 & 0.58 & 0.29 \\
\hline & & $12.43-15.26$ & $0-9$ & $0-2$ & $0-2$ & $0-1$ & $0-2$ & $0-1$ \\
\hline \multirow{2}{*}{ Smokers } & \multirow{2}{*}{47} & $14.32 \pm 0.58^{\mathrm{a}}(14.30)$ & $3.36^{\mathrm{a}}$ & 0.79 & 0.04 & 0.23 & $1.06^{\mathrm{a}}$ & $0.53^{\mathrm{a}}$ \\
\hline & & $13.09-15.55$ & $0-13$ & $0-4$ & $0-1$ & $0-1$ & 0.5 & $0-2.5$ \\
\hline \multirow{2}{*}{ S-F } & \multirow{2}{*}{29} & $14.23 \pm 0.54(14.16)$ & 2.12 & 0.69 & 0.04 & 0.27 & 1.00 & 0.50 \\
\hline & & $13.31-15.42$ & $0-12$ & $0-2$ & $0-1$ & $0-1$ & $0-2$ & $0-1$ \\
\hline \multirow{2}{*}{ S-M } & \multirow{2}{*}{47} & $14.43 \pm 0.62(14.53)$ & 4.90 & 0.90 & 0.05 & 0.19 & 1.14 & 0.57 \\
\hline & & $13.09-15.55$ & $0-13$ & $0-4$ & $0-1$ & $0-1$ & $0-5$ & $0-2.5$ \\
\hline AGE (years) & & & & & & & & \\
\hline 20-20 & 30 & $13.68 \pm 0.92(13.65)$ & 1.87 & 0.53 & 0.07 & 0.07 & 0.67 & 0.33 \\
\hline $20-29$ & 30 & $12.43-15.55$ & $0-12$ & $0-2$ & $0-2$ & $0-1$ & $0-2$ & $0-1$ \\
\hline & & $14.09 \pm 0.79(14.26)$ & 2.86 & 0.54 & 0.03 & 0.22 & 0.78 & 0.39 \\
\hline $30-39$ & 37 & $12.10-15.27$ & $0-10$ & $0-2$ & $0-1$ & $0-1$ & $0-2$ & $0-1$ \\
\hline & & $14.20 \pm 0.65(14.24)$ & 2.90 & 0.76 & 0.07 & $0.31^{\mathrm{b}}$ & 1.14 & 0.57 \\
\hline $40-49$ & 29 & $12.67-15.04$ & $0-13$ & $0-4$ & $0-1$ & $0-1$ & $0-5$ & $0-2.5$ \\
\hline & & $14.10 \pm 0.64(14.03)$ & 2.39 & 0.78 & 0.06 & 0.06 & 0.89 & 0.44 \\
\hline $50-64$ & 18 & $12.52-15.10$ & $0-9$ & $0-2$ & $0-1$ & $0-1$ & $0-2$ & $0-1$ \\
\hline TIME OF BLOOD & ING & & & & & & & \\
\hline Winter (I-III) & 29 & $14.13 \pm 0.88(14.24)$ & $3.55^{\mathrm{c}}$ & 0.55 & 0.07 & 0.10 & 0.72 & 0.36 \\
\hline Winter (I-III) & 29 & $12.10-15.55$ & $0-12$ & $0-2$ & $0-2$ & $0-1$ & $0-2$ & $0-1$ \\
\hline & & $14.03 \pm 0.80(14.11)$ & 2.33 & 0.54 & 0.08 & 0.17 & 0.79 & 0.39 \\
\hline Spring (IV-VI) & 52 & $12.43-15.10$ & $0-13$ & $0-2$ & $0-1$ & $0-1$ & $0-2$ & $0-1$ \\
\hline & & $13.56 \pm 0.65(13.65)$ & 0.58 & 0.58 & & 0.21 & 0.79 & 0.39 \\
\hline Summer (VII-IX) & 19 & $12.54-14.44$ & $0-2$ & $0-2$ & - & $0-1$ & $0-2$ & $0-1$ \\
\hline & & $14.34 \pm 0.50^{c}(14.29)$ & $3.86^{\mathrm{c}}$ & $1.21^{\mathrm{d}}$ & & 0.29 & $1.50^{\mathrm{e}}$ & $0.75^{\mathrm{e}}$ \\
\hline Autumn (X-XII) & 14 & $13.56-15.04$ & $0-9$ & $0-4$ & - & $0-1$ & $0-5$ & $0-2.5$ \\
\hline All subjects & & $14.01 \pm 0.79(14.05)$ & 2.54 & 0.63 & 0.05 & 0.18 & 0.86 & 0.43 \\
\hline All subjects & 114 & $12.10-15.55$ & 0-13 & $0-4$ & $0-2$ & $0-1$ & $0-5$ & $0-2.5$ \\
\hline
\end{tabular}

F, female subject; $M$, male subject; NS, non-smoker; S, smoker; $X$, diagnostic exposure to X-rays; MRI, diagnostic exposure to magnetic resonance imaging; $\mathrm{AD}$, antirheumatic drugs; $\mathrm{LTN}$, long tailed nuclei; $\mathrm{CA}$, chromosome aberrations; $\mathrm{B}_{1}$, chromatid break $\mathrm{B}_{2}$, chromosome break; Ac, acentric fragment. Multiple comparisons were made using multifactor ANOVA with post-hoc Scheffé test; significantly increased values $(\mathrm{P}<0.05)$ were: ${ }^{a}$ to non-smokers; ${ }^{b}$ with regard to other subgroups based on the age; ${ }^{c}$ with regard to samples collected in spring and summer; ${ }^{\mathrm{d}}$ with regard samples collected in spring and winter; ${ }^{\mathrm{e}}$ with regard samples collected in winter.

Distribution of subjects with respect to the extent of DNA migration in their white blood cells is displayed on Fig. 4, where differences between whole group $v s$. subgroups with or without medicinal exposure are shown.

\section{Chromosome aberration test}

Individual results on the frequencies of chromosome aberrations (CA) recorded in peripheral blood lymphocytes (PBL) are summarized in Table 1 , while comparisons of group mean values are reported in Tables 2 and 3.

As reported in Table 1, an inter-individual diversity among the subjects studied was observed. Individual values for the total number of CA in PBL were in the range of $0-5$ CA per 200 cells, with an average of 0.96 CA per 200 cells. Total percentage of aberrant cells was in the range of $0-2.5 \%$, with an average of $0.48 \%$. Only three types of structural 
chromosome aberrations were observed. The most frequent aberration type were chromatid breaks. Their incidence was determined as a mean frequency of 0.68 per 200 cells. The mean yield of acentric fragments was 0.18 per 200 cells, while chromosome breaks were determined with a mean frequency of 0.11 per 200 cells (Table 2).

All comparisons between subgroups were made by multifactor ANOVA with post-hoc Scheffé test. As reported in Table 2, smoking habit significantly influenced the total number as well as the percentage of CA recorded in PBL. Although male subjects had a little higher total number of CA compared to female subjects, this difference was not statistically significant (Table 2). Age significantly influenced the total number of acentric fragments only in PBL of subjects aged between 40 and 49 years (Table 2). Diagnostic X-ray exposure significantly enhanced total number of chromosome breaks, total number of structural CA in PBL, as well as percentage of aberrant cells. The effects of other medical exposures should be carefully evaluated because in the present study only two subjects reported medicinal exposure to MRI, or occasional intake of antirheumatic drugs.

Moreover, significant variations between total number of CA and the percentage of aberrant cells recorded in blood samples collected in different seasons were observed. The highest total number of CA and the percentage of aberrant cells were recorded in blood samples taken in autumn and in winter (Table 2). However, statistical analysis confirmed that they were mostly influenced by diagnostic Xray exposure and smoking habits.

The results of the separate study of the subpopulation without medical exposure $(n=114)$ are reported in Table 3. They confirmed that smoking significantly influences the total number as well as the percentage of CA recorded in PBL. Age also significantly influenced the total number of acentric fragments in PBL of subjects aged between 40 and 49 years (Table 3 ). Significantly increased incidence of chromatid breaks, total number of CA and the percentage of aberrant cells were recorded in blood samples taken in autumn, but they were mostly influenced by smoking habit (Table 3).

The results of statistical analyses showed that the total number of chromatid breaks, chromosome breaks and acentric fragments were in a positive correlation with the total number of CA as well as with the total percentage of aberrant cells.

Moreover, they indicate that a positive correlation also exists between the increased DNA migration (expressed both as mean tail length and total number of LTN) and the total number of CA, as well as with the total percentage of aberrant cells.

\section{DISCUSSION}

Assessment of normal levels of DNA damage in the general population is essential for the proper interpretation of data obtained by monitoring of populations occupationally or accidentally exposed to known or potentially genotoxic agents. Although many biomonitoring studies indicate that the baseline genetic damage in white blood cells is affected by various endogenous and external factors, it is not clear how an individual's inborn genetic constitution may influence the yield of such damage.

Over the years our laboratory has accumulated a lot of data on cytogenetic biomarkers and alkaline comet assay in general and various exposed human populations (Kašuba et al., 1995; Garaj-Vrhovac et al., 1997; 1999; Garaj-Vrhovac, 1999; Rozgaj et al., 1999; Garaj-Vrhovac \& Kopjar, 2000; 2003; Kopjar \& Garaj-Vrhovac, 2001; Rozgaj et al., 2001; Želježić \& Garaj-Vrhovac, 2001; 2002; Kašuba et al., 2002). However, from time to time the values of these biomarkers have to be re-evaluated, especially for the general population, to establish the upper level of normal variability so that positive effect of exposure can be more readily recognized.

In the study presented here, two different biomarkers, one of exposure (the alkaline comet assay), and the other of effect (chromosome aberration test) were used to evaluate the baseline DNA damage in white blood cells of healthy blood donors randomly selected from the Croatian general population. The main aim of this study was to investigate the association between the values of the two biomarkers and several external and internal factors, as well as their mutual relationships.

Our goal was to investigate a common "healthy" population. For that reason, prior to the final selection of subjects who participated in the study, we intentionally excluded subjects with any infection, chronic disease or cancer history as well as those with known exposure to confounding factors such as high alcohol consumption, vitamin and antibiotic intake as well as subjects involved in intensive sportive activities.

In spite of the very rigorous procedures (i.e. exactly the same conditions of all steps of the procedures and a very good reproducibility of the assays employed), we observed an inter-individual variability among the subjects studied. Variability is a typical feature of biological systems, extensively reported by various authors when using the comet assay and cytogenetic endpoints (Anderson et al., 1993; Betti et al., 1994; Kašuba et al., 1995; Hellman et al., 1997; Wojewódzka et al., 1998; Landi et al., 1999; Stephan \& Pressl, 1999; Morillas et al., 2002; GarajVrhovac \& Kopjar, 2003). 
Heterogeneity in the level of DNA damage recorded in our study could be in part attributed to individual genome sensitivity. The DNA damage detected by alkaline comet assay represents a steady state between induction of lesions and their repair. Therefore a low damage level as assessed experimentally in an individual may be the result of an actual low number of lesions or of a high efficiency of repair (Somorovská et al., 1999; Wojewódzka et al., 1999).

Although in many subjects a positive correlation between increased DNA migration and total number of structural chromosome aberrations was observed, it was difficult to explain some individual cases, where such a correlation between both biomarkers did not exist. This observation raises the general question of the relationship between the induction of DNA damage in resting lymphocytes, and its subsequent fixation in genetic alterations after stimulation. When considered together, our data suggest that DNA damage induced in vivo in circulating lymphocytes can be largely repaired, thus escaping fixation, as has been also reported earlier (Betti et al., 1994; Andreoli et al., 1999; Mayer et al., 2002). As we observed, the levels of DNA damage detected by chromosome aberration analysis in some subjects could be low, or there would be no detectable increase in cytogenetic biomarkers, even in the presence of a sizeable amount of primary DNA damage recorded in vivo. These observations can be easily explained if we consider that the data of the comet assay were based on responses in white blood cells, while the data gathered in the cytogenetic tests are obtained exclusively with proliferation-stimulated lymphocytes. Because white blood cells are a heterogeneous mixture of cells, as regarding their life-span and sensitivity, some differences may be due to different cell populations being compared. Despite the risk of reduced sensitivity, we prefer the use of whole blood samples for the alkaline comet assay. Many other investigators also report the use of whole leukocyte fractions or whole blood when studying induced or basal levels of DNA damage in the comet assay. The use of whole blood is easier and avoids the possibility of inducing additional (e.g. oxidative) DNA damage during the process of separating the various cell types from each other, which in many cases will be hard to control for (Hellman et al., 1997; Speit et al., 2003).

The difference between the results in the comet assay and the cytogenetic tests is basically due to variations in the type of DNA alterations that the test system detects: the comet assay detects repairable DNA lesions or alkali-labile sites while cytogenetic tests detect fixed mutations which persist for at least one mitotic cycle (Kassie et al., 2000). Many of primary induced DNA lesions are successfully re- paired in a few minutes (4-15 min) (Tice, 1995) to a couple of hours (2-3 h) (Singh et al., 1988) after infliction. However, the increased levels of primary DNA damage in some subjects could be also attributed to endogenous factors, especially intracellular oxidative stress pronounced after exposure to genotoxic agents (for example tobacco smoke or diagnostic X-rays). This could give an increased steadystate DNA damage, high enough to be detected by the sensitive comet assay. DNA modification might give rise to alkali labile sites that are converted into single strand breaks during alkaline electrophoresis. On the other hand, if base damage is located close together ( $<10 \mathrm{bp}$ apart) on opposite DNA strands, simultaneous excision of such modified bases can lead to the formation of DSB, the supposed initial lesion in the formation of chromosomal aberrations (CA). Incompletely repaired or unrepaired DSB are converted into chromosome and chromatid breaks (Pfeiffer et al., 2000) that may be visualized on metaphase preparations.

Biomonitoring studies with a combination of cytogenetic tests and comet assay are of special interest because they enable comparison of the relative sensitivity of the two test systems and may also give a clue about the fraction of DNA damage detected in the comet assay that will lead to fixed mutations (Kassie et al., 2000). As a biomarker, the comet assay reflects the current exposure (over the previous few weeks) and the actual levels of DNA damage present in white blood cells at the moment of blood sampling. Cytogenetic biomonitoring, on the other hand, provides additional information on the DNA damage levels, especially on past exposures. $\mathrm{Hu}-$ mans are exposed to a variety of natural or synthetic genotoxic substances, able to modify the baseline levels of DNA damage. During the life-time everyone "accumulates" some level of radiation exposure, mainly due to background radiation in the environment, diagnostic exposures, or small amounts of radioisotopes ingested with food. From the studies on subjects occupationally exposed to ionizing radiation it is known that the consequence of an in vivo exposure to ionizing radiation also might be complex unstable chromosome aberrations, such as dicentric or ring chromosomes (Bender et al., 1988a; Natarajan, 1993; Hagelström et al., 1995). However, these aberration types were not observed in our study; on the contrary, most of chromosome aberrations detected in peripheral blood lymphocytes were of chromatid-type. Such aberration type is mostly induced by chemical mutagens and carcinogens in lymphocytes during S-phase or post-replicative stages (Bender et al., 1988a; Natarajan, 1993).

The results on the effects of different confounding factors obtained in the present study are in good agreement with previous observations. 
Taken together, sex and age did not significantly influence the levels of primary DNA damage and incidence of structural chromosome aberration in white blood cells. Preceding biomonitoring studies with the comet assay and chromosome aberrations reported contradictory data on the effects of gender. Some authors observed differences between male and female subjects (Anderson et al., 1988; Betti et al., 1994; Wojewódzka et al., 1998; Mendoza-Núñez et al., 2001; Bajpayee et al., 2002), while others found similar levels of primary DNA damage in both sexes (Bender et al., 1988b; Anderson et al., 1993; Bonassi et al., 1995; Kašuba et al., 1995; Frenzilli et al., 1997; Major et al., 1998; Pitarque et al., 1999; Stephan \& Pressl, 1999; Zhu et al., 1999). In our study female and male subjects had comparable levels of primary DNA damage and structural chromosome aberrations in their white blood cells. Careful evaluation of the data showed that the higher number of long tailed nuclei (LTN), as observed in male subjects, was caused mainly by smoking habit and diagnostic X-ray exposure. This observation was confirmed when subjects with any kind of medical exposure were excluded from the assessment.

Previous biomonitoring studies with the comet assay and chromosome aberrations also reported contradictory data on possible age-related increase of DNA damage in white blood cells. Some authors reported age-related increase of primary DNA damage as detected by the alkaline comet assay (Singh et al., 1991; Piperakis et al., 1998; Moretti et al., 2000; Maluf et al., 2001; Mendoza-Núñez et al., 2001). Based on the observations of other authors, the age of the individual appears to have no significant effect on the mean basal level of DNA damage (Betti et al., 1994; Frenzilli et al., 1997; Awara et al., 1998; Wojewódzka et al., 1998; Palus et al., 1999; Pitarque et al., 1999; Zhu et al., 1999; 2001). A similar situation was observed in our study. Previously reported data on the age-related incidence of chromosome aberrations are also contradictory (Bolognesi et al., 1997). While some authors did not find a significant increase of the frequency of chromosome aberration with age (Anderson et al., 1988; 1993; Kašuba et al., 1995), others reported significant age dependency of the number of acentric fragments in peripheral blood lymphocytes (Bender et al., 1988b; Stephan \& Pressl, 1999). Our results are in good agreement with those observations. The restricted number of subjects older than 50 years included in our study makes it impossible to draw a firm conclusion about an association between the age and the number and incidence of distinct types of structural CA in their lymphocytes. Nevertheless, it is noteworthy that the incidence of chromatid breaks, acentric fragments and corresponding number of total CA gradually increases with age in subjects aged from 20 to 49 years, and this increase was statistically significant for acentric fragments in subgroup aged 40-49 years as compared to other subgroups analyzed.

The values of both biomarkers studied indicate that the most effective confounding factors that modulate the baseline damage in white blood cells were smoking habit and medical exposure. The data on the smoking-induced DNA damage in biomonitoring studies are still controversial. Some studies clearly confirmed the genotoxic effects of tobacco smoke, as well as its modifying effect of genome damage induced by other agents (Betti et al., 1994; Frenzilli et al., 1997; Piperakis et al., 1998; Palus et al., 1999; Stephan \& Pressl, 1999; Zhu et al., 1999; 2001; Hininger et al., 2004), while others reported no significant increase of the values of the biomarkers evaluated (Anderson et al., 1988; 1993; Bender et al., 1988b; Kašuba et al., 1995; Šrám et al., 1998; Pitarque et al., 1999; Wojewódzka et al., 1999; Maluf et al., 2001; Speit et al., 2003; Hoffman \& Speit, 2005). It is likely that the design of study as well as differences in other lifestyle factors contribute to the inconsistent observations. In our investigation smokers represent $45 \%$ of the population studied, and smoking was found to be a confounding factor independently of other endogenous or external factors. Evaluation of the comet assay data indicates that smokers had significantly increased levels of primary DNA damage and a higher number of long tailed nuclei as compared to non-smokers. Moreover, the observed increases were in a positive correlation with the total number of chromosome aberrations and the percentage of cells with aberrations. We also confirmed the modifying effect of smoking on the genome damage in white blood cells of subjects with diagnostic exposure to X-rays.

Diagnostic exposure to X-rays was also found to be a confounding factor that significantly enhanced the levels of DNA damage in white blood cells of subjects involved in our study. Although many investigators usually exclude subjects with diagnostic X-ray exposure from their studies, we decided to evaluate their baseline DNA damage. In this research $30 \%$ of subjects were exposed to diagnostic X-rays (chest or dental) up to one month prior to the study, mostly during the pre-employment medical check-ups. Although many authors reported that the doses of radiation used in medical diagnosis are relatively low - the dose of radiation from a chest X-ray is about $0.020-0.025 \mathrm{mSv}$, while dental X-ray exposes a subject to doses ranged about 5 $\mu \mathrm{Sv}$ to $0.03 \mathrm{mSv}$ (Butler et al., 1985; Benke, 1995; Rehani, 2000; Berrington De González \& Darby, 2004) - they might increase the baseline levels of DNA damage, as detectable with the sensitive alkaline comet assay. This assumption was confirmed in our study where subjects with diagnostic X-ray exposure 
had slightly increased DNA migration and a higher number of LTN as compared to subjects without any medicinal exposure. Moreover, increased levels of primary DNA damage were in a positive correlation with the total number of chromosome aberrations in peripheral blood lymphocytes as well as with the total number of cells with CA. Diagnostic X-ray exposure in healthy subjects was also in a positive correlation with the incidence of chromosome breaks in peripheral blood lymphocytes. Despite the increased DNA migration and elevated incidence of structural chromosome aberration in peripheral lymphocytes of subjects who reported medicinal exposure to MRI, or occasional intake of antirheumatic drugs, the effects of these medical exposures should be carefully evaluated because the number of subjects who reported them was too small.

Some authors reported seasonal variation in the results of the comet assay, with more damage detected in samples obtained during summer months (Betti et al., 1995; Frenzilli et al., 1997; Møller et al., 1998; 2000; 2002). However, we were not able to confirm those observations. When the whole group was studied, the highest primary DNA damage (expressed both as DNA migration and the number of LTN) was recorded in samples collected in winter. However, careful statistical evaluation indicated that this was mostly influenced by medicinal X-ray exposure and smoking habits. Namely, the majority of blood samples of subjects with diagnostic Xray exposure, and a lot of samples of smokers were collected in winter. When subjects with any kind of medical exposure were excluded from the study, the highest primary DNA damage was recorded in samples collected in autumn. In these samples we found significantly increased DNA migration and the number of LTN, as well as an increased incidence of chromatid breaks, total number of CA as well as the percentage of cells with CA. However, it is difficult to say whether the DNA damaging effects could be accounted by sunlight exposure only because during the autumn the prevalence of samples donated by smokers was also observed. One of the main reasons why we did not find a significant modifying effect of sunlight exposure was the small number of blood samples analysed during the summer season, while the second was the prevalence of samples donated by non-smokers throughout this season.

Despite their limitations, the results obtained in the present study revealed a lot of background data that may be of value in future genotoxicological monitoring in the Republic of Croatia. They also confirmed that the alkaline comet assay and chromosome aberration test are sensitive biomarkers that have to be further evaluated and standardized for the assessment of DNA damage in human bio- monitoring studies as well as in cases of accidental exposures.

\section{Acknowledgement}

The authors gratefully acknowledge the cooperation of all the volunteers who participated in this study. We wish to thank Mrs. Snježana Ramić for excellent technical assistance and Dr. Višnja Oreščanin for statistical advice.

This investigation was supported by the Croatian Ministry of Science, Education and Sports (grant No. 0022020).

\section{REFERENCES}

Albertini RJ, Anderson D, Douglas GR, Hagmar L, Hemminki K, Merlo F, Natarajan AT, Norppa H, Shuker DEG, Tice R, Waters MD, Aitio A (2000) ICPS guidelines for the monitoring of genotoxic effects of carcinogens in humans. Mutat Res 463: 111-172.

Anderson D, Jenkinson PC, Dewdney RS, Francis AJ, Godbert P, Butterworth KR (1988) Chromosome aberrations, mitogen-induced blastogenesis and proliferative rate index in peripheral lymphocytes from 106 control individuals of the U.K. population. Mutat Res 204: 407420.

Anderson D, Francis AJ, Godbert P, Jenkinson PC, Butterworth KR (1993) Variability in chromosome aberrations, sister chromatid exchanges, and mitogen-induced blastogenesis in peripheral lymphocytes from control individuals. Environ Health Perspect 104: (suppl 3) 83-88.

Andreoli C, Rossi S, Leopardi P, Crebelli R (1999) DNA damage by hydroquinone in human white blood cells: analysis by alkaline single-cell gel electrophoresis. $\mathrm{Mu}$ tat Res 438: 37-45.

Awara WM, El-Nabi SH, El-Gohary M (1998) Assessment of vinyl chloride-induced DNA damage in lymphocytes of plastic industry workers using a single cell gel electrophoresis technique. Toxicology 128: 9-16.

Bajpayee M, Dhawan A, Parmar D, Kumar Pendey A, Mathur N, Seth PK (2002) Gender-related differences in basal DNA damage in lymphocytes of a healthy Indian population using the alkaline comet assay. Mutat Res 520: 83-91.

Bender MA, Awa AA, Brooks AL, Evans HJ, Groer PO, Littlefield LG, Pereira C, Preston J, Wachholz BW (1988a) Current status of cytogenetic procedures to detect and quantify previous exposure to radiation. Mutat Res 196: 103-159.

Bender MA, Preston J, Leonard RC, Pyatt BE, Gooch PC, Shelby MD (1988b) Chromosomal aberration and sister chromatid exchange frequencies in peripheral lymphocytes of a large human population sample. Mutat Res 204: 421-433.

Benke KK (1995) Biological effects of low-level radiation and diagnostic medical X-rays J Aust Coll Nutr Environ Med 14: 17-20.

Berrington de González A, Darby S (2004) Risk of cancer from diagnostic X-rays: estimates for the UK and 14 other countries. Lancet 363: 345-351.

Betti C, Davini T, Giannessi L, Loprieno N, Barale R (1994) Microgel electrophoresis assay (comet test) and SCE 
analysis in human lymphocytes from 100 normal subjects. Mutat Res 307: 323-333.

Betti C, Davini T, Giannessi L, Loprieno N, Barale R (1995) Comparative studies by comet assay and SCE analysis in human lymphocytes from 200 healthy subjects. $M u$ tat Res 343: 201-207.

Bolognesi C, Abbondandolo A, Barale R, Casalone R, Dalprà L, De Ferrari M, Degrassi F, Forni A, Lamberti L, Lando C, Migliore L, Padovani P, Pasquini R, Puntoni R, Sbrana I, Stella M, Bonassi S (1997) Age-related increase of baseline frequencies of sister chromatid exchanges, chromosome aberrations and micronuclei in human lymphocytes. Cancer Epidemial Biomarkers Prev 6: 249-256.

Bonassi S, Bolognesi C, Abbondandolo A, Barale R, Bigatti $\mathrm{P}$, Camurri L, Dalprà L, De Ferrari M, Forni A, Lando C, Padovani P, Pasquini R, Stella M, Puntoni R (1995) Influence of sex on cytogenetic end points: evidence from a large human sample and review of the literature. Cancer Epidemial Biomarkers Prev 4: 671-679.

Butler PF, Conway BJ, Suleiman OH, Koustenis GH, Showalter CK (1985) Chest radiography: a survey of techniques and exposure levels currently used. Radiology 156: 533-536.

Chuan C-H, Hu M-L (2004) Use of whole blood directly for single-cell gel electrophoresis (comet) assay in vivo and white blood cells for in vitro assay. Mutat Res 564: $75-82$.

Collins AR (2004) The comet assay for DNA damage and repair: principles, applications, and limitations. Mol Biotechnol 26: 249-261.

Frenzilli G, Betti C, Davini T, Desideri M, Fornai E, Giannessi L, Maggiorelli F, Paoletti P, Barale R (1997) Evaluation of DNA damage in leukocytes of ex-smokers by single cell gel electrophoresis. Mutat Res 375: 117-123.

Garaj-Vrhovac V (1999) Micronucleus assay and lymphocyte mitotic activity in risk assessment of occupational exposure to microwave radiation. Chemosphere 39: 2301-2312.

Garaj-Vrhovac V, Kopjar N (2000) Cytogenetic monitoring of cardiology unit hospital workers exposed to ultrasound. J Appl Toxicol 20: 259-264.

Garaj-Vrhovac V, Kopjar N (2003) The alkaline comet assay as biomarker in assessment of DNA damage in medical personnel occupationally exposed to ionizing radiation. Mutagenesis 18: 263-271.

Garaj-Vrhovac V, Fučić A, Kubelka D, Hebrang A (1997) Assessment of genome damage in occupational exposure to ionizing radiation and ultrasound. Mutat Res 395: 101-105.

Garaj-Vrhovac V, Kopjar N, Besendorfer V, Papeš D (1999) Induction of micronuclei in human lymphocytes after occupational exposure to ultrasound. Chemosphere 38: 3541-3553.

Hagelström AH, Gorla NB, Larippa IB (1995) Chromosomal damage in workers occupationally exposed to chronic low level ionizing radiation. Toxicol Lett 76: 113-117.

Hagmar L, Bonassi S, Strömberg U, Mikoczy Z, Lando C, Hansten I-L, Huici Montagud A, Knudsen L, Norppa $\mathrm{H}$, Reuterwall C, Tinnerberg H, Brøgger A, Forni A, Högstedt B, Lambert B, Mitelman F, Nordenson I, Salomaa S, Skerfving S (1998) Cancer predictive value of cytogenetic markers used in occupational health surrveilance programs: a report from an ongoing study by the European Study Group on Cytogenetic Biomarkers and Health. Mutat Res 405: 171-178.

Hellman B, Vaghef H, Friis L, Edling C (1997) Alkaline single cell gel electrophoresis of DNA fragments in biomonitoring for genotoxicity: an introductory study on healthy human volunteers. Int Arch Occup Environ Health 69: 185-192.

Hellman B, Friis L, Vaghef H, Edling C (1999) Alkaline single cell gel electrophoresis and human biomonitoring for genotoxicity: a study on subjects with residential exposure to radon. Mutat Res 442: 121-132.

Hininger I, Chollat-Namy A, Sauvagio S, Osman M, Faure H, Cadet J, Favier A, Rousell A-M (2004) Assessment of DNA damage by comet assay on frozen total blood: method and evaluation in smokers and non-smokers. Mutat Res 558: 75-80.

Hoffman H, Speit G (2005) Assessment of DNA damage in peripheral blood of heavy smokers with the comet assay and the micronucleus test. Mutat Res 581: 105-114.

International Atomic Energy Agency (2001) International Atomic Agency Technical Report Series 405, Cytogenetic Analysis for Radiation Dose Assessment (Vienna: IAEA).

Kassie F, Parzefall W, Knasmüller S (2000) Single cell gel electrophoresis assay: a new technique for human biomonitoring studies. Mutat Res 463: 13-31.

Kašuba V, Šentija K, Garaj-Vrhovac V, Fučić A (1995) Chromosome aberrations in peripheral blood lymphocytes from control individuals. Mutat Res 346: 187-193.

Kašuba V, Rozgaj R, Kopjar N, Želježić D, Jazbec A (2002) Sister chromatid exchange analysis in peripheral blood lymphocytes of healthy individuals. Biologia 57: 401407.

Kopjar N, Garaj-Vrhovac V (2001) Application of the alkaline comet assay in human biomonitoring for genotoxicity: a study on Croatian medical personnel handling antineoplastic drugs. Mutagenesis 16: 71-78.

Landi S, Frenzilli G, Milillo PC, Cocchi L, Sbrana I, Scapoli C, Barale R (1999) Spontaneous sister chromatid exchange and chromosome aberration frequency in humans: the familial effect. Mutat Res 444: 337-345.

Major J, Jakab MG, Tompa A (1998) Genotoxicological monitoring of 175 subjects living in the green belts, inner town of near chemical industrial estates in Greater Budapest agglomeration, Hungary. Mutat Res 412: 9-16.

Maluf SW, Ferreira Passos D, Bacelar A, Speit G, Erdtman B (2001) Assessment of DNA damage in lymphocytes of workers exposed to X-radiation using the micronucleus test and the comet assay. Environ Mol Mutagen 38: 311-315.

Mastaloudis A, Yu T-W, O'Donnel RP, Frei B, Dashwood RH, Traber MG (2004) Endurance exercise results in DNA damage as detected by the comet assay. Free Radical Biol Med 36: 966-795.

Mayer C, Popanda O, Zelezny O, von Brevern MC, Bach A, Bartsch H, Schmetzer P (2002) DNA repair capacity after $\gamma$-irradiation and expression profiles of DNA repair genes in resting and proliferating human peripheral blood lymphocytes. DNA Repair 1: 237-250.

Mendoza-Núñez VM, Sánchez-Rodríguez MA, RetanaUgalde R, Vargas-Guadarrama LA, Altamirano-Lozano MA (2001) Total antioxidant levels, gender, and age as risk factors for DNA damage in lymphocytes of the elderly. Mech Ageing Dev 122: 835-847.

Møller P, Knudsen LE, Frentz G, Dybdahl M, Wallin H, Nexø BA (1998) Seasonal variation of DNA damage and repair in patients with non-melanoma skin cancer and referents with and without psoriasis. Mutat Res 407: 25-34.

Møller P, Knudsen LE, Loft S, Wallin H (2000) The comet assay as rapid test in biomonitoring occupational exposure to DNA-damaging agents and effect of con- 
founding factors. Cancer Epidemial Biomarkers Prev 9: 1005-1015.

Møller P, Wallin H, Holst E, Knudsen LE (2002) Sunlightinduced DNA damage in human mononuclear cells. FASEB J 16: 45-53.

Moretti M, Villarini M, Scassellati Sforzilini G, Pasquini R (2000) Pesticide-induced primary DNA damage in peripheral blood leukocytes of farm workers evaluated by the computerized "comet" assay. Biomarkers 5: 192-204.

Morillas MJ, Guillamet E, Surralles J, Creus A, Marcos R (2002) Spontaneous and induced genetic damage in $T$ lymphocyte subsets evaluated by the comet assay. $\mathrm{Mu}$ tat Res 514: 39-48.

Natarajan, A. T (1993) Mechanisms for induction of mutations and chromosome alterations. Environ Health Persp 101: (suppl 3) 225-229.

Natarajan AT, Boei JJ, Darroudi F, Van Diemen PCM, Dulout F, Hande MP, Ramalho AT (1996) Current cytogenetic methods for detecting exposure and effects of mutagens and carcinogens. Environ Health Perspect 104: (suppl 3) 445-248.

Palus J, Dziubałtowska E, Rydzyński K (1999) DNA damage detected by the comet assay in the white blood cells of workers in a wooden furniture plant. Mutat Res 444: 61-74.

Pfeiffer P, Goedecke W, Obe G (2000) Mechanisms of DNA double strand break repair and their potential to induce chromosomal aberrations. Mutagenesis 15: 289-302.

Piperakis SM, Visvardis EE, Sagnou M, Tassiou AM (1998) Effects of smoking and aging on oxidative DNA damage of human lymphocytes. Carcinogenesis 19: 695-698.

Pitarque M, Vaglenov A, Nosko M, Hirvonen A, Norppa H, Creus A, Marcos R (1999) Evaluation of DBA damage by the comet assay in shoe workers exposed to toluene and other organic solvents. Mutat Res 441: 115-127.

Rehani MM (2000) CT: caution on radiation dose. Ind J Radiol Imaging 10: 19-20.

Rojas E, Lopez MC, Valverde M (1999) Single cell gel electrophoresis assay: methodology and applications. J Chromatogr B 722: 225-254.

Rozgaj R, Kašuba V, Šentija K, Prlić I (1999) Radiation-induced chromosomal aberrations and haematological alterations in hospital workers. Occup Med 49: 353-360.

Rozgaj R, KašubaV, Jazbec A (2001) Preliminary study of cytogenetic damage in personnel exposed to anesthetic gases. Mutagenesis 16: 139-143.

Şardaş S, Aygün N, Gamli M, Ünal Y, Berk N, Karakaya AE (1998) Use of alkaline comet assay (single cell gel electrophoresis technique) to detect DNA damages in lymphocytes of operating room personnel occupationally exposed to anaestethic gases. Mutat Res 418: 93100.

Singh NP (2000) Microgels for estimation of DNA strand breaks, DNA protein crosslinks and apoptosis. Mutat Res 455: 111-127.

Singh NP, Mc Coy MT, Tice RR, Schneider EL (1988) A simple technique for quantitation of low levels of DNA damage in individual cells. Exp Cell Res 175: 184-191.

Singh NP, Danner DB, Tice RR, Pearson JD, Brant LJ, Morrell CH, Schneider EL (1991) Basal DNA damage in in- dividual human lymphocytes with age. Mutat Res 256: $1-6$.

Somorovská M, Szabová E, Vodička P, Tulinská J, Barančoková M, Fábry R, Liškova A, Riegerová Z, Petrovská H, Kubová J, Rausová K, Dušinská M, Collins AR (1999) Biomonitoring of genotoxic risk in workers in a rubber factory: comparison of the comet assay with cytogenetic methods and immunology. Mutat Res 445: 181-192.

Speit G, Witton-Davies T, Heepchantree W, Trenz K, Hoffman $H$ (2003) Investigations on the effect of cigarette smoking in the comet assay. Mutat Res 542: 33-42.

Stephan G, Pressl S (1999) Chromosomal aberration in peripheral lymphocytes from healthy subjects as detected in first cell division. Mutat Res 446: 231-237.

Šrám RJ, Podrazilová K, Dejmek J, Mračková G, Pilčík T (1998) Single cell gel electrophoresis assay: sensitivity of peripheral white blood cells in human population studies. Mutagenesis 13: 99-103.

Tice RR (1995) The single cell gel / comet assay: a microgel electrophoretic technique for the detection of DNA damage and repair in individual cells. In Environmental Mutagenesis (Phillips DH, Vennit S, eds), pp 315-339 Oxford, Bioscientific.

Tice RR, Agurell E, Anderson D, Burlinson B, Hartmenn A, Kobayashi H, Miyamae Y, Rojas E, Ryu JC, Sasaki YF (2000) Single cell gel/comet assay: guidelines for in vitro and in vivo genetic toxicology testing. Environ Mol Mutagen 35: 206-121.

Valverde M, Ostrosky-Wegman P, Rojas E, Fortoul E, Meneses MNF, Ramírez M, Díaz-Barriga F, Cebrian M (1999) The application of single cell gel electrophoresis or comet assay to human monitoring studies. Salud Públ Méx 41: 109-113.

Wojewódzka M, Kruszewski M, Iwanenko T, Collins AR, Szumiel I (1998) Application of the comet assay for monitoring DNA damage in workers exposed to chronic low-dose irradiation. I. Strand breakage. Mutat Res 416: 21-35.

Wojewódzka M, Kruszewski M, Iwanenko T, Collins AR, Szumiel I (1999) Lack of adverse effect of smoking habit on DNA strand breakage and base damage, as revealed by the alkaline comet assay. Mutat Res 440: 19-25.

Zhu CQ, Lam TH, Jiang CQ, Wei BX, Lou X, Liu WW, Lao XQ, Chen YH (1999) Lymphocyte DNA damage in cigarette factory workers measured by the comet assay. Mutat Res 444: 1-6.

Zhu CQ, Lam TH, Jiang CQ (2001) Lymphocyte DNA damage in bus manufacturing workers. Mutat Res 491: 173-181.

Želježić D, Garaj-Vrhovac V (2001) Chromosomal aberration and single-cell gel electrophoresis (comet) assay in the longitudinal risk assessment of occupational exposure to pesticides. Mutagenesis 16: 359-363.

Želježić D, Garaj-Vrhovac V (2002) Sister chromatid exchange and proliferative rate index in the longitudinal risk assessment of occupational exposure to pesticides. Chemosphere 46: 295-303. 